TI 2014-112/II

Tinbergen Institute Discussion Paper
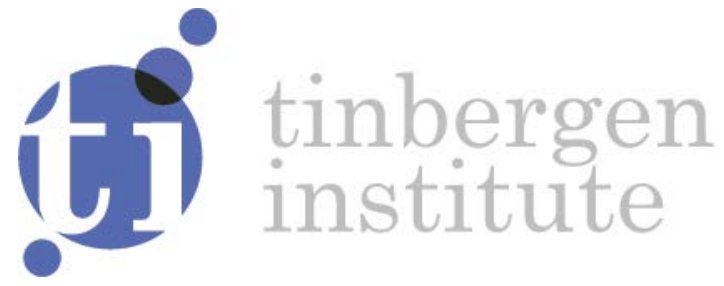

\title{
Research among Copycats: R\&D, Spillovers, and Feedback Strategies
}

Revision: J une 22, 2017

Grega Smrkolj ${ }^{1}$

Florian Wagener ${ }^{2}$

${ }^{1}$ Newcastle University, UK

${ }^{2}$ University of Amsterdam and Tinbergen Institute, The Netherlands 
Tinbergen Institute is the graduate school and research institute in economics of Erasmus University Rotterdam, the University of Amsterdam and VU University Amsterdam.

Contact: discussionpapers@tinbergen.nl

More TI discussion papers can be downloaded at http://www.tinbergen.nl

Tinbergen Institute has two locations:

Tinbergen Institute Amsterdam

Gustav Mahlerplein 117

1082 MS Amsterdam

The Netherlands

Tel.: + $31(0) 205984580$

Tinbergen Institute Rotterdam

Burg. Oudlaan 50

3062 PA Rotterdam

The Netherlands

Tel.: +31(0)10408 8900 


\title{
Research among Copycats: R\&D, Spillovers, and Feedback Strategies*
}

\author{
Grega Smrkolj ${ }^{\dagger} \quad$ Florian Wagener ${ }^{\ddagger}$
}

June, 2017

\begin{abstract}
We study a stochastic dynamic game of process innovation in which firms can initiate and terminate $\mathrm{R} \& \mathrm{D}$ efforts and production at different times. We discern the impact of knowledge spillovers on the investments in existing markets, as well as on the likely structure of newly forming markets, for all possible asymmetries between firms. While an increase in spillovers may improve the likelihood of a competitive market, it may at the same time reduce the level to which a technology is developed. We show that the relation between spillovers, R\&D efforts, and surpluses depends on relative as well as absolute efficiency of firms. High spillovers are not necessarily pro-competitive as they can make it harder for the laggard to catch up with the technology leader.
\end{abstract}

Keywords: Process innovation, R\&D, Spillovers, Differential game, Feedback Nash equilibrium JEL: C63, C73, D43, D92, L13, O31

\section{Introduction}

Contemporary markets are flooded with imitations - it is hard to find a business model, good, or service that is not a variation or an adaptation of some earlier version. Dell and HP are only two out of many firms that cloned IBM's Personal Computer. Atari's video game attracted as many as 75 imitators, led by Nintendo. More recently, Samsung's lawyers could not tell the difference between Samsung's Galaxy Tab and Apple's iPad in court. ${ }^{1}$ While more latent than product imitations, imitations of business processes abound as well and often even transcend the sector in which they were first introduced. Walmart's automated supply chain management strategies were imitated by its competitors (e.g., Kmart, Tesco),

\footnotetext{
${ }^{*}$ The authors would like to thank Chaim Fershtman, Joe Harrington, Jeroen Hinloopen, Peter Kort, Juan Mañez, Jose L. Moraga, Sander Onderstal, Randolph Sloof, and Adriaan Soetevent, as well as seminar participants at the Tinbergen Institute Organizations \& Markets Seminar (Amsterdam, January 2013), at the 8th Royal Economic Society Postgraduate Presentation Meeting (London, January 2013), at the 11th International Industrial Organization Conference (Boston, May 2013), at the 19th International Conference of the Society for Computational Economics (Vancouver, July 2013), at the Newcastle University Business School (Newcastle upon Tyne, January 2014), at the 16th International Symposium on Dynamic Games and Applications (Amsterdam, July 2014), at the 68th European Meeting of the Econometric Society (Toulouse, August 2014), at the Annual Conference of the Royal Economic Society (Manchester, March 2015), at the 49th Annual Conference of Canadian Economic Association (Toronto, May 2015), at the Australian Conference of Economists (Brisbane, July 2015), and at the 42nd Annual Conference of the European Association for Research in Industrial Economics (Munich, August 2015) for valuable comments and discussions.

${ }^{\dagger}$ Newcastle University Business School. Corresponding author. Address: Newcastle University Business School, 5 Barrack Road, Newcastle upon Tyne, NE1 4SE, UK; grega.smrkolj@newcastle.ac.uk.

${ }^{\ddagger}$ University of Amsterdam and Tinbergen Institute; f.o.o.wagener@uva.nl.

${ }^{1}$ Sam Biddle, "Even Samsung's Lawyers Can't Tell the Difference Between Its Tablet and an iPad", Gizmodo, October 14, 2011, accessed August 2, 2012, http://gizmodo.com/5849803/even-samsung-cant-tell-the-difference-between-its-tablet-andipad.
} 
but also by companies in other sectors, such as Ryanair. South West Airlines' innovative business model which led to the low cost revolution in air travel was successfully imitated by both Ryanair and easyJet. Henry Ford's introduction of the moving assembly line did not only reduce the cost of his Model T car, but also revolutionized manufacturing processes across industries worldwide. Today a similar revolution is taking place as e-commerce adopted by various sorts of organizations is reducing the need for paperwork and the pace of imitation seems ever growing. Shenkar (2010) even writes about the "imovation challenge" - companies that want to succeed, need to fuse innovation and imitation as in the future it will not be possible anymore "to rely on innovation or imitation alone to drive competitive advantage." Moreover, Bloom et al. (2013) recently found large knowledge spillovers for a panel of US firms. Consequently, they estimate that social returns to $R \& D$ are between two and three times the private returns.

In this paper, we develop a continuous-time dynamic model in which two competing firms need to decide how much to produce and how much to invest in cost-reducing R\&D over an infinite horizon. We then study how information leakages, or spillovers, affect industry dynamics and structure through their impact on innovation incentives of firms at different stages of development. ${ }^{2}$ Our focus is process innovation, interpreted as any improvement in "the way things are done" that enables a firm to satisfy a given consumer need at lower cost. This focus is motivated by the fact that product innovations often cannot be successfully introduced in the market without accompanying process innovations ${ }^{3}$ and that over time relative productivity becomes decisive for surviving in the market. In fact, a higher emphasis on process innovation by Japanese companies is believed to be one of the main reasons for their increasing competitiveness over their American counterparts (Bhoovaraghavan et al., 1996).

We solve for a feedback Nash equilibrium of the differential game, which is characterized by a system of highly nonlinear implicit partial differential equations, by a variant of the numerical method of lines (Schiesser, 1991): by discretizing technology levels, but not time, the system of partial differential equations is approximated by a system of ordinary differential equations. The solution to this system is then obtained by standard methods.

The seminal contribution to the analysis of firms' strategic R\&D decisions in the presence of spillovers is d'Aspremont and Jacquemin (1988). In their two-stage model, firms first invest in cost-reducing R\&D and then play a Cournot game in the product market. While the amount of R\&D done by each firm tends to decrease as spillovers increase (due to a free-riding effect), equilibrium costs, industry output, and welfare increase as long as the level of spillovers is below the critical value of 0.5 . While here spillovers occur at the level of R\&D outcomes, in the model of Kamien et al. (1992) they occur at the level of R\&D inputs (see Amir (2000) for a detailed discussion of differences in the two modelling approaches). In this case, increases in spillovers worsen technological performance in the competitive equilibrium for all levels of spillovers. The two models fostered numerous generalizations and extensions. Surveying early contributions, De Bondt (1997) concludes that "some, but not too high barriers to imitation" seems to be most conducive to innovative activity.

\footnotetext{
${ }^{2}$ Hardly any business idea is immune to imitation. As Arrow (1962, p. 615) explains, "The very use of the information in any productive way is bound to reveal it, at least in part. Mobility of personnel among firms provides a way of spreading information. Legally imposed property rights can provide only a partial barrier, since there are obviously enormous difficulties in defining in any sharp way an item of information and differentiating it from similar sounding items."

${ }^{3} \mathrm{~A}$ good example is the modern plasma display, the concept of which was first conceived at the University of Illinois in 1964. At that time, it was too expensive to mass produce using the existing technology. It took several years for IBM to launch a 48-cm monochrome plasma display destined for commercial use in 1983. Several additional years of research and improvements on the licensed technology of first innovators were needed for Fujitsu to present the first 53-cm fully-colored hybrid display in 1992. Philips' plasma display claimed to be the first to be presented to the retail sector on a large scale in 1997 at a price of no less than $\$ 14,999$. Later, Pioneer, Sony, LG, Samsung, and a few others also entered this market. Thanks to subsequent improvements in the technology and concomitant reductions in the production costs, different variants of plasma TVs are nowadays available for less than $\$ 1,000$.
} 
Recently, continuous-time dynamic models have emerged. ${ }^{4}$ Their advantage over static models is that in them firms can smooth their investment over a long time, like usually observed in practice. In the model of Cellini and Lambertini (2009), which is a dynamic version of d'Aspremont and Jacquemin (1988), both firms start with the same level of marginal costs and invest continuously in cost-reducing R\&D. This investment gradually reduces initial costs towards the steady-state level. In sharp contrast to its static counterparts, their model leads to a lower level of equilibrium costs for any increase in spillovers. Smrkolj and Wagener (2016) however recently pointed out that, unlike previously believed, the equilibrium in Cellini and Lambertini (2009) is not subgame perfect, such that the paper effectively discusses only the open-loop situation, in which firms commit to the entire investment schedule at the beginning of the game and therefore cannot respond to each other's actions in the course of time. Our current paper fills the gap in the literature by providing the feedback solution — which by construction is subgame perfect - to a differential game that shares its fundamental setup with Cellini and Lambertini (2009). It improves on that and other related papers in several dimensions which we discuss below; most importantly, our setup captures the feature that $R \& D$ investments in process innovations can be made long before production is viable. This is different from the well-known patent race mechanism.

Recent contributions in the field of differential games emphasize the importance of initial conditions for long-run outcomes. For instance, in Dawid et al. (2015), a decision of an established incumbent to invest in risky $R \& D$ to extend its product range depends on its initial product capacity and knowledge stock. In Hinloopen et al. (2013), the value of the initial marginal cost determines whether a monopolist develops a technology further or exits the market. In particular, it can be optimal for a firm to invest in process innovation before entering the product market if the initial cost is above the reservation or 'choke' price, that is, the lowest price at which the quantity sold is zero. This differs vastly from the related static and dynamic models with spillovers (e.g., Cellini and Lambertini (2009), Petit and Tolwinski (1999)) which all assume that the initial cost is below the choke price, thereby imposing the coexistence of production and R\&D at all times. These models contradict the observation that for many new technologies research starts long before production, and are silent on situations in which initial production costs of a new technology exceed the highest willingness to pay in the market. Hence, the existence of a market and an $R \& D$ process is already assumed and the question left is how spillovers affect the (size of) $R \& D$ efforts. These investigations are local in nature: they discuss (near-) steady-state behavior and concern themselves with comparative statics questions of the sort "What is the influence of changing this parameter on that steady-state quantity?" We call these 'questions of degree'.

Also of considerable interest are 'questions of kind': "How does changing this parameter affect in which steady state the system will end up?" Clearly, to answer such questions, out-of-steady-state dynamics has to be considered. A distinguishing feature of our approach is that it allows for a firm to optimally decide to never enter a particular market ${ }^{5}$ or to exit some existing market in due time. Consequently, we are able to analyze not only how spillovers affect the investments on existing markets, but also how they influence the likelihood that a new market will be formed, and if so, how its likely structure - monopoly or duopoly - relates to the level of spillovers, and this so for every possible combination of firms' initial unit costs. Our framework therefore puts conclusions of the previous literature, typically holding only for a subset of possible cost levels, into a broader perspective.

Hinloopen et al. (2013) are the first to provide a global analysis for a continuous-time dynamic model

\footnotetext{
${ }^{4}$ See Doraszelski and Judd (2012) for a discussion of substantial advantages of continuous-time formulations over their discrete-time counterparts in dynamic stochastic games.

${ }^{5}$ Elmer Bolton, a scientist-manager at DuPont, one of the most innovative corporations in American business history, was famous for saying to the company's chemists who in his opinion often lacked the awareness that the success of the company depends on its products being commercially exploitable: “This is very interesting chemistry, but somehow I don't hear the tinkle of the cash register" (Hounshell, 1988).
} 
of R\&D. The present article extends their dynamic framework in two significant directions. First, rather than a monopoly, we consider a differential game with two competing firms, which are both free to enter the market. Second, given that innovation is inherently uncertain, we introduce stochasticity to the R\&D process.

At the outset of the game, each firm has an initial unit cost of production $c_{i}(0)$, corresponding to the initial level of a particular technology. This value may differ between firms, for instance if the imitator lacks the production experience of the innovator. Firms can increase their production efficiency by exerting R\&D efforts, which in turn are subject to imitation. Higher production efficiency in turn makes them stronger competitors on the Cournot product market. Firms' product market participation constraints are taken into account explicitly. Consequently, the R\&D process and production do not need to coexist at all times and firms can enter or exit the product market and initiate or cease their R\&D processes at different times. In the present set-up, the initial unit production cost of a firm is allowed to be above the choke price; in such a situation, it is optimal for a firm to postpone production. The R\&D costs have to be made that will only be recuperated in the future, when the technology has been advanced to the point that production is viable. The outlays for the bulk of $R \& D$ may precede the revenues. This is the key feature that our model can capture and that is inaccessible to static or near-steady-state approaches.

The empirical literature indicates large differences in productivity across firms due to their differences in information technology and management practices (see, e.g., Baily et al. (1992), Bloom and Van Reenen (2011)). Due to their prominence in practice, we put asymmetries between firms and their dynamic evolvement to the center of our investigation. It has been a long-standing indication in the literature, confirmed in a dynamic, though deterministic, framework by Petit and Tolwinski (1999), that higher spillovers can prevent the monopolization of the industry by easing imitation. Using a richer framework and considering all possible absolute and relative differences in firms' production costs, this paper shows that while intuitive, the above result is not universally true. We show that high spillovers can also make market monopolization possible, sometimes even more so than lower spillovers. At relatively lower unit costs of the leader, additional R\&D efforts benefit the leader progressively less and the laggard progressively more, and the incentives of the leader to exert R\&D efforts can be rather low. This makes it harder for the laggard to catch up with the leader. Notably, the ability to copy is not worth much when there is little to copy. Consequently, smaller cost asymmetries can suffice to induce the monopolization of the industry at larger spillovers. We also show that the relation between spillovers and welfare is more involved than previously thought - it is non-monotonic and affected by both the relative and absolute initial position of firms.

Our paper is also related to a large literature on innovation and imitation that has developed in the field of endogenous growth theory. Among recent contributions, Lucas Jr and Moll (2014) study productivity growth resulting from agents in an economy dividing their time between production and imitation. In Benhabib et al. (2014) agents make a portfolio choice between investment in imitation and innovation. In the equilibrium, agents close enough to the technological frontier focus on innovation, while others follow a balanced growth path of imitators. Similarly, in König et al. (2016) firms make an endogenous choice between in-house R\&D and imitation. In each case, the outcome is random and independent of invested efforts. Firms at the technological frontier choose innovation over imitation as they are less likely to find more efficient firms to imitate. The reverse is true for firms far from the frontier. Firms studied are technology leaders from different sectors, each monopolizing their own sector. As such the authors abstract from strategic competition within industries to study the productivity distribution of firms across industries.

Furukawa (2007) studies intellectual property rights (IPR) and their effects on economic growth. While stronger IPR protection increases incentives to innovate, it at the same time increases the share of 
monopolized sectors in the economy. The result is an U-shaped relationship between IPR and growth, where both too strong and too week protection hurts innovation. Acemoglu and Akcigit (2012) conclude that the optimal intellectual property rights policy is state dependent in the sense that greater protection should be given to those technological leaders that are further ahead. The reason is that such a policy incentivizes also firms with a limited technological lead to innovate more, as besides making them more productive, further innovation now also grants them additional protection.

While these papers explore some dimensions we do not consider, none of them studies the effect of knowledge spillovers on strategic competition within an industry. In König et al. (2016) firms never innovate and imitate at the same time, in Benhabib et al. (2014) firms in the equilibrium segment into innovators and imitators, and in Acemoglu and Akcigit (2012) a follower can copy the technology frontier only after the expiration of the patent. In contrast to them, in our model, knowledge spillovers regularly occur at the level of firms' $R \& D$ outputs, such that, much like in practice, imitation is concomitant with innovation and both technology leaders and followers can benefit from within-industry spillovers. As our model allows for a technology to never be developed and for firms to exit the market, we are also able to study the effect of spillovers, and thus imitation, on market formation and structure. This is not the case in König et al. (2016) or Acemoglu and Akcigit (2012), where some sector's product is always offered by that sector's technology leader.

While Acemoglu and Akcigit (2012) suggest a relatively stricter IPR protection at later stages of development, our model leads to a vastly different conclusion. ${ }^{6}$ We find strong IPR protection (low knowledge spillovers) potentially most conducive to $R \& D$ at the early stages of development. As low spillovers make it difficult to catch up if a firm falls behind, they can induce firms to invest a lot when each is trying to secure its position in the future product market. When a technology is relatively developed, low spillovers in our model usually lead to the lowest total surplus. They are especially conducive to market monopolization if market profitability at later stages of development is comparably low and so any large catch-up investment, potentially needed to prevent market monopolization, hardly profitable.

The remainder of the paper is organized as follows. Section 2 describes the model specification. Section 3 explains our computational approach to obtaining a feedback Nash equilibrium solution. Section 4 discusses equilibrium strategies and corresponding industry dynamics. Section 5 considers welfare effects of spillovers. Section 6 summarizes and concludes.

\section{Model}

The dynamic game is defined in continuous time and over an infinite horizon: $t \in[0, \infty)$. There are two firms that potentially compete in a market for a homogenous good with demand

$$
p(t)=\max \left\{A-q_{i}(t)-q_{j}(t), 0\right\},
$$

where $p(t)$ is the market price, $q_{i}(t)$ is the quantity produced by firm $i=\{1,2\}, q_{j}(t)$ is the quantity produced by its rival $(i \neq j)$, and $A$ is the choke price (the lowest price at which the quantity sold is zero). We thus assume that consumers are static and do not make any intertemporal decisions. Likewise, the state of demand is known to firms. In our model, the source of uncertainty for firms is technology and not demand. This simple formulation of the product market allows for a rich modeling of firms' choices regarding production and $\mathrm{R} \& \mathrm{D}$ investment. ${ }^{7}$

\footnotetext{
${ }^{6}$ Of course a question remains to what an extent is the IPR policy able to affect within-industry knowledge spillovers.

${ }^{7}$ To have consumers that are forward looking and firms that form expectations about the future behavior of consumers is an important, but challenging, extension to our model that we leave for future work.
} 
At the outset of the game, each firm obtains an exogenous technology, represented by its unit cost level $c_{i}(0)$. For simplicity, we assume that firms may differ in their production cost, being identical in every other aspect. While both firms produce with constant returns to scale, each firm can reduce its unit cost $c_{i}(t)>0$ by investing in R\&D. This process is subject to spillovers. Firm $i$ exerts R\&D effort $k_{i}(t) \geq 0$ and as a consequence of these investments, its unit cost (state variable) evolves over time according to

$$
\frac{\mathrm{d} c_{i}}{c_{i}}=\left(-k_{i}-\beta k_{j}+\delta\right) \mathrm{d} t+\sigma_{i} \mathrm{~d} B_{i},
$$

where $k_{j}=k_{j}(t), j \neq i$, is the R\&D effort exerted by the rival, $\beta \in[0,1]$ is a degree of spillovers, and $\delta>0$ reflects the constant rate of efficiency reduction due to the aging of technology and organizational forgetting (Besanko et al., 2010; Hinloopen et al., 2013). ${ }^{8}$ Moreover, the $B_{i}(t)$ are standard Wiener processes or Brownian motions, $d B_{i} \sim \mathcal{N}(0, d t)$, with $\sigma_{i}>0$ denoting their strength. Equation (2) is of the form $\mathrm{d} c=\mu(c, k) \mathrm{d} t+\sigma(c) \mathrm{d} B$, where $\mu$ and $\sigma$ are drift and diffusion, respectively, of a controllable Itô process $c$ (see Kloeden and Platen, 1992). Hence, firms face some randomness in the evolution of their unit costs (random discoveries, mechanical failures, strikes and changes in factor prices occur regularly in business practice). The random variations in $c$ are high when $c$ is high, i.e. when the technology is not fully developed yet and the firm is still relatively inexperienced. Furthermore, the processes $B_{i}$ are assumed to be independent and of equal strength, that is $\sigma_{1}=\sigma_{2}=\sigma \cdot{ }^{9}$ In other words, both firms face the same level of technological uncertainty.

Note that Equation (2) is not linear in state and controls, and that consequently the game is not linear-quadratic. Observe in (2) that the smaller the $c_{i}$, the smaller the effect of particular $k_{i}$ on $\dot{c}_{i}$. Further innovations require increasingly more $R \& D$ efforts. Exerting $R \& D$ effort is costly. This cost equals $b k_{i}(t)^{2}$ per unit of time, where $b>0$ is inversely related to the cost-efficiency of the R\&D process. In assuming decreasing returns to $\mathrm{R} \& \mathrm{D}$, we follow the bulk of the literature (see, e.g., d'Aspremont and Jacquemin (1988), Kamien et al. (1992), Qiu (1997), or Hinloopen et al. (2013)). Low values of $\beta$ correspond to strong intellectual property protection and the ability of firms to prevent involuntary leaks of information. The reverse is true for high values of $\beta$. We treat the value of $\beta$ as given for firms. ${ }^{10}$ Both firms discount the future with the same constant rate $r>0$. The instantaneous profit of firm $i$ is:

$$
\pi_{i}(t)= \begin{cases}\left(A-q_{i}(t)-q_{j}(t)-c_{i}(t)\right) q_{i}(t)-b k_{i}(t)^{2} & \text { if } p(t)>0 \\ -c_{i}(t) q_{i}(t)-b k_{i}(t)^{2} & \text { if } p(t)=0\end{cases}
$$

yielding its expected total discounted profits over time:

$$
\Pi_{i}=\mathbb{E}_{0} \int_{0}^{\infty} \pi_{i}(t) e^{-r t} d t
$$

\footnotetext{
${ }^{8}$ In the model of Besanko et al. (2010), a firm's marginal cost depends on its stock of know-how, which can be eroded if gains from learning are less than losses from organizational forgetting due to labor turnover, periods of inactivity and failure to institutionalize tacit knowledge. Hinloopen et al. (2013) further reason that a firm sluggish in its R\&D will find it more difficult to identify and assimilate knowledge from the environment and also more costly to incorporate complementary inputs, which are subject to inherent evolution and typically purchased, in its production process. Empirical studies also support a positive depreciation rate. For instance, Benkard (2000) estimates that in a production of wide-bodied airframes the stock of know-how depreciates at the rate of $4 \%$ per month.

${ }^{9}$ Other correlation structures could also be analyzed using our methods. To investigate how asymmetric outcomes are, or are not, generated by asymmetric initial conditions, we need to investigate a model in which firms are as symmetric as possible, in order that we may be sure that asymmetric outcomes are only the result of an asymmetry in initial conditions. Symmetric Wiener processes, together with other symmetry restrictions on parameters, serve to this end.

${ }^{10}$ In general, $\beta$ may be one of a firm's strategic variables. See Katsoulacos and Ulph (1998) and Amir et al. (2003) for an attempt to endogenize the degree of spillovers. Von Hippel (1988) provides empirical evidence for firms being consensually involved in information sharing. See also Shenkar (2010). Amir et al. (2000) allow for spillovers to differ between firms.
} 
here and below, $\mathbb{E}_{t}$ is the expectation operator conditional on information available up to time $t$.

\subsection{Rescaling}

Our model depends on six parameters: $A, b, \delta, r, \beta$, and $\sigma$. Some of these are mathematically redundant: by choosing the measurement scale of units appropriately, it turns out that the resulting model depends effectively only on four parameters.

Lemma 1. After choosing measurement units of variables and parameters appropriately, the state equations and discounted profits read as

$$
\mathrm{d} c_{i}=\left(1-\left(k_{i}+\beta k_{j}\right) \phi\right) c_{i} \mathrm{~d} t+c_{i} \sqrt{2 \varepsilon} \mathrm{d} B_{i}
$$

and

$$
\Pi_{i}=\mathbb{E}_{0} \int_{0}^{\infty} \pi_{i}(t) e^{-\rho t} d t
$$

respectively. These depend on the parameter $\beta$ as well as on the new parameters $\phi=A /(\delta \sqrt{b}), \rho=r / \delta$, and $\varepsilon=\sigma^{2} / 2 \delta$.

Proof. See Appendix A.

The new parameter $\phi$ introduced by the scaling transformation (cf. Lemma 1 in Hinloopen et al. (2013), Lemma 2.1 in Hinloopen et al. (2017)) effectively substitutes for $A, b$, and $\delta$. It captures the profit potential of a technology. Higher potential revenues come with a higher $A$, and each unit of R\&D effort costs more if $b$ increases, while it reduces unit cost by less the higher is $\delta$. In sum, a lower (higher) $\phi$ corresponds to a lower (higher) profit potential. The new parameter $\rho$ is a rescaled discount rate and $\varepsilon$ is a measure of volatility. They have similar interpretations as former $r$ and $\sigma$, respectively.

We emphasize that by performing a scaling transformation — also known as 'nondimensionalization'; see Fowler (1997, Ch. 2) - we do not restrict ourselves to any particular parameter values, nor do we lose any property of the model (e.g., equilibria). Each choice of parameters in the original model corresponds to a choice of parameters in the rescaled model; the complexity reduction obtained by scaling is due to the fact that many different parameterizations of the original model give rise to mathematically equivalent models.

For instance, if the efficiency reduction rate $\delta$ equals 0.1 per annum in the original parametrization, the fact that it equals 1 in the rescaled model only means that a time interval of length 1 in the rescaled model corresponds to a time interval of 10 years in the original model. By choosing the time scale appropriately, we reduce the number of free parameters affecting the solution, and we thereby simplify the exploration of the parameter space. Once we have obtained a solution to the rescaled model, we obtain the solution to the original model by applying the conversion equations specified in Appendix A.

\subsection{Product market and equilibrium output levels}

Firms compete in a product market by strategically setting their output levels. The analysis of this market is simplified by the fact that production, unlike $R \& D$ efforts, does not influence the evolution of the unit cost levels. Hence, the firms play a Cournot duopoly game with respect to production at each instant of time, and the output levels, while depending on unit costs, $q_{i}=\psi_{i}\left(c_{1}, c_{2}\right)$, are static Cournot-Nash equilibria of the instantaneous game. 
Proposition 1. The strategy profile $\psi^{*}\left(c_{1}, c_{2}\right)=\left(\psi_{1}^{*}\left(c_{1}, c_{2}\right), \psi_{2}^{*}\left(c_{1}, c_{2}\right)\right)$, where

i) $\psi_{i}^{*}\left(c_{1}, c_{2}\right)=\frac{1-2 c_{i}+c_{j}}{3}$ if $2 c_{1}-c_{2}<1, \quad 2 c_{2}-c_{1}<1$;

ii) $\quad \psi_{i}^{*}\left(c_{1}, c_{2}\right)=0, \quad \psi_{j}^{*}\left(c_{1}, c_{2}\right)=\frac{1-c_{j}}{2} \quad$ if $\quad 2 c_{i}-c_{j} \geq 1, \quad c_{j}<1$;

iii) $\quad \psi_{1}^{*}\left(c_{1}, c_{2}\right)=0, \quad \psi_{2}^{*}\left(c_{1}, c_{2}\right)=0 \quad$ if $\quad c_{1} \geq 1, \quad c_{2} \geq 1$,

is a Cournot-Nash equilibrium of a quantity setting duopoly in the product market $(i, j \in\{1,2\}, i \neq j)$.

These equilibria are illustrated in Figure 1, which is drawn in a logarithmic scale to match the later presentation of numerical results. The curves $E_{1}$ and $E_{2}$ are the 'entry/exit' curves for firms 1 and 2, respectively: to the left of $E_{1}$, firm 1 is not active in the product market; below $E_{2}$, firm 2 is not active. The two curves divide state space into four regions: 'Duopoly', 'Monopoly of Firm $i$ ', for $i=1$ or 2 , and 'No production'.

Both firms produce positive amounts only for combinations of unit costs in the 'Duopoly' region. There, the market price is higher than the unit cost of each firm (the first case in the above proposition), and firm $i$ earns a profit of

$$
g_{i}\left(c_{1}, c_{2}\right)=\frac{\left(1-2 c_{i}+c_{j}\right)^{2}}{9} .
$$

In the region 'Monopoly of Firm 1', the market price is lower than firm 2's unit cost; firm 2 therefore optimally sells nothing. Firm 1 earns there a monopoly profit

$$
g_{1}\left(c_{1}, c_{2}\right)=\frac{\left(1-c_{1}\right)^{2}}{4} .
$$

The roles are reversed in the region 'Monopoly of Firm 2'.

Finally, in the 'No production' region, the unit costs of both firms are higher than the choke price $(A=1)$, and as firms could sell a positive amount only at negative mark-ups, neither firm produces. Firms can reach the production region by reducing their unit costs through $R \& D$ investment.

In sum, the sales profit of firm $i$ is given by

$$
g_{i}\left(c_{1}, c_{2}\right)=\left\{\begin{array}{llll}
\left(1-2 c_{i}+c_{j}\right)^{2} / 9 & \text { if } & 2 c_{1}-c_{2}<1, & 2 c_{2}-c_{1}<1, \\
\left(1-c_{i}\right)^{2} / 4 & \text { if } & 2 c_{j}-c_{i} \geq 1, & c_{i}<1, \\
0 & & \text { otherwise. }
\end{array}\right.
$$

The function $g_{i}$ is continuous. The total instantaneous profit is the sales profit $g_{i}$ diminished by the R\&D expenditure $k_{i}^{2}$ :

$$
\pi_{i}=g_{i}\left(c_{1}, c_{2}\right)-k_{i}^{2} .
$$

The substitution of equilibrium output levels in firms' profit functions has resulted in the profit function of firm $i$ being dependent only on unit costs and R\&D effort. Consequently, the problem of the firms is reduced to finding optimal $R \& D$ efforts. 


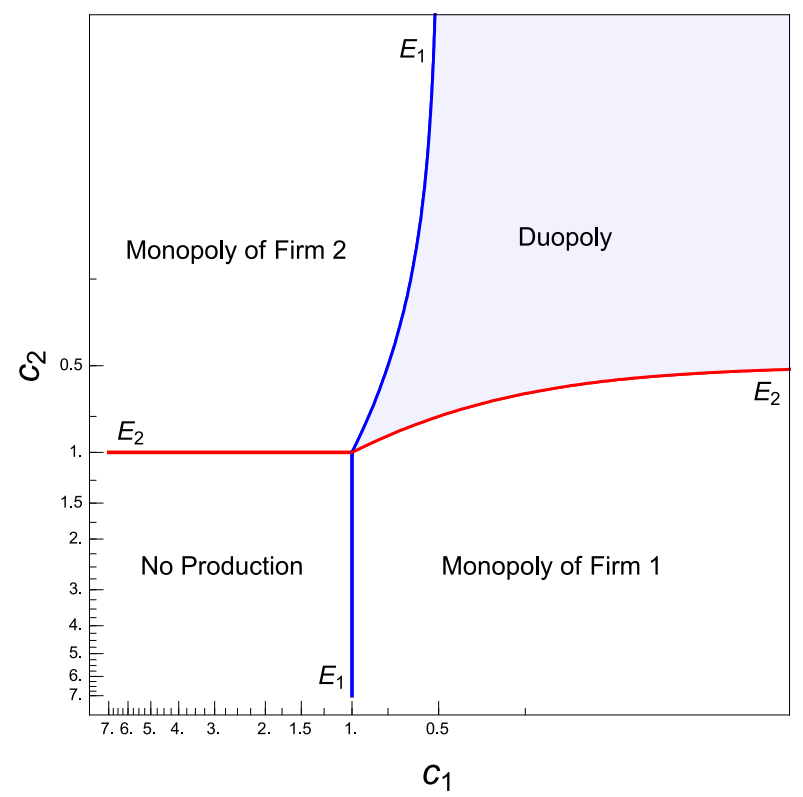

Figure 1: Product-market activity.

\subsection{Problem statement}

To sum up formally, in the two-firm differential game, each firm tries to maximize its expected pay-off

$$
\Pi_{i}=\mathbb{E}_{0} \int_{0}^{\infty}\left[g_{i}\left(c_{1}, c_{2}\right)-k_{i}^{2}\right] e^{-\rho t} d t
$$

through its choice of $\mathrm{R} \& \mathrm{D}$ effort $k_{i} \geq 0$, subject to the state equations

$$
\mathrm{d} c_{i}=\left(1-\left(k_{i}+\beta k_{j}\right) \phi\right) c_{i} \mathrm{~d} t+c_{i} \sqrt{2 \varepsilon} \mathrm{d} B_{i}, \quad i=1,2,
$$

where $c_{i}>0$ for $i=1,2$.

\subsection{Equilibrium}

In the game, each firm tries to maximize its total discounted profits by selecting a strategy which specifies its action, that is its R\&D effort, at each instant of time. In the stochastic context, it is most natural to consider feedback strategies, where the actions are given as functions of the current state of the system (see Başar and Olsder, 1999). The corresponding feedback Nash equilibria are characterized by a dynamic programming equation. The resulting equilibrium strategies are strongly time consistent or 'subgame perfect'. Here, the state of the game is determined by the pair $\left(c_{1}, c_{2}\right)$ of unit production costs of the firms, and a strategy for firm $i$ is a function $k_{i}=\Gamma_{i}\left(c_{1}, c_{2}\right)$, specifying its $\mathrm{R} \& \mathrm{D}$ effort $k_{i}$ as a function of the state.

In this context, a 'feedback Nash equilibrium' is a pair $\left(\Gamma_{1}^{*}, \Gamma_{2}^{*}\right)$ of feedback strategies, such that the choice

$$
k_{i}(t)=\Gamma_{i}^{*}\left(t, c_{1}(t), c_{2}(t)\right)
$$

for all $t \geq t_{0}$ maximizes the present discounted value of firm $i$ 's profits, given that the other firm chooses 
its R\&D level by (16) with $i$ replaced by $j$. This implies that choosing $k_{i}$ according to (16) maximizes

$$
\mathbb{E}_{t_{0}} \int_{t_{0}}^{\infty} \pi_{i}\left(k_{i}, \Gamma_{j}^{*}\left(t, c_{1}, c_{2}\right)\right) e^{-\rho t} d t
$$

subject to the state equations. Introduce the value $W^{i}=W^{i}\left(t_{0}, c_{1,0}, c_{2,0}\right)$ at $c_{i, 0}=c_{i}\left(t_{0}\right), i=1,2$, as the value of the integral. Then $W^{i}$ is the maximum discounted value of profits at time $t=t_{0}$ that can be earned by firm $i$, given that the other firm pursues its equilibrium strategy. If $\Gamma^{*}$ is a feedback Nash equilibrium solution to our dynamic game, there exist functions $W^{i}$, satisfying the so-called HamiltonJacobi-Bellman equations (see Başar and Olsder, 1999, Ch. 6.5) either everywhere, or at least in the sense of viscosity solutions (Crandall and Lions, 1983):

$$
\begin{aligned}
-W_{t}^{i}=\varepsilon c_{1}^{2} W_{c_{1} c_{1}}^{i}+ & \varepsilon c_{2}^{2} W_{c_{2} c_{2}}^{i} \\
+\max _{k_{i} \geq 0}[ & \left(g_{i}\left(c_{1}, c_{2}\right)-k_{i}^{2}\right) e^{-\rho t}+W_{c_{i}}^{i}\left(1-\left(k_{i}+\beta \Gamma_{j}^{*}\left(t, c_{1}, c_{2}\right)\right) \phi\right) c_{i} \\
& \left.+W_{c_{j}}^{i}\left(1-\left(\Gamma_{j}^{*}\left(t, c_{1}, c_{2}\right)+\beta k_{i}\right) \phi\right) c_{j}\right],
\end{aligned}
$$

where $i \in\{1,2\}, i \neq j$. We adopt the convention that a subscript to the value function indicates a partial derivative of that function with respect to each subscripted variable. This equation is complemented by the asymptotic terminal condition ${ }^{11}$

$$
\lim _{t \rightarrow \infty} W^{i}\left(t, c_{1}, c_{2}\right)=0
$$

Introduce the current-time value function $V^{i}\left(t, c_{1}, c_{2}\right)$ by setting $W^{i}=V^{i} e^{-\rho t}$. That is, $V^{i}$ equals the profits earned when firm $i$ starts in the state $\left(c_{1}, c_{2}\right)$ at time $t$ and invests optimally, while firm $j$ pursues its equilibrium strategy. Equation (18) can then be written as

$$
\begin{aligned}
\rho V^{i}-V_{t}^{i}=\varepsilon c_{1}^{2} V_{c_{1} c_{1}}^{i}+ & \varepsilon c_{1}^{2} V_{c_{2} c_{2}}^{i} \\
+\max _{k_{i} \geq 0}[ & g_{i}\left(c_{1}, c_{2}\right)-k_{i}^{2}+V_{c_{i}}^{i}\left(1-\left(k_{i}+\beta \Gamma_{j}^{*}\left(t, c_{1}, c_{2}\right)\right) \phi\right) c_{i} \\
& \left.+V_{c_{j}}^{i}\left(1-\left(\Gamma_{j}^{*}\left(t, c_{1}, c_{2}\right)+\beta k_{i}\right) \phi\right) c_{j}\right] .
\end{aligned}
$$

These equations are complemented by the following analogue of Equation (19)

$$
\lim _{t \rightarrow \infty} V^{i}\left(t, c_{1}, c_{2}\right) e^{-\rho t}=0 .
$$

If the value functions $V^{i}$ are continuously differentiable, equations (20) hold everywhere. If this assumption is not valid, which may typically happen for $\varepsilon=0$, the $V^{i}$ do not satisfy the equations (20) in a classical sense. In such a case, these equations are understood in the sense of viscosity solution (see Crandall and Lions, 1983). If $V^{i}$ is differentiable at some $\left(t, c_{1}, c_{2}\right)$, then

$$
\Gamma_{i}^{*}\left(t, c_{1}, c_{2}\right)=\max \left\{-\frac{1}{2} \phi\left(V_{c_{i}}^{i} c_{i}+\beta V_{c_{j}}^{i} c_{j}\right), 0\right\} .
$$

\footnotetext{
${ }^{11}$ Condition (19) is a standard transversality condition in the literature (see, e.g., Prop. 7.1 in Haurie et al. (2012) or Thm. 4 in Benveniste and Scheinkman (1982)). We can write $W\left(0, c_{1,0}, c_{2,0}\right)=\mathbb{E}_{0}\left[\int_{0}^{T} \pi(u) e^{-\rho u} d u+W\left(T, c_{1, T}, c_{2, T}\right)\right], T \rightarrow \infty$. Condition (19) says that the expected net present value of profits at infinity (the last term) must be zero. That is, profits do not grow unboundedly large. For an optimal solution, all specific contributions to the objective function have to be made in finite time and not in some very distant future.
} 
This relation can be used to eliminate the $\Gamma_{i}^{*}$ from equations (20). The result is a coupled system of parabolic partial differential equations for the value functions $V^{i}, i=1,2$. For a single firm, under deterministic cost dynamics $(\varepsilon=0)$ there are situations where the value function is not differentiable over the entire space (Hinloopen et al., 2013), as 'indifference' or 'Skiba' points appear for certain parameter values. These are points where a firm is indifferent between developing and not developing a technology further. At such a point, the optimal investment function is multiple valued, and the corresponding value function is kinked. We expect the same phenomenon to occur in the case of two competing firms in the deterministic limit $\varepsilon \rightarrow 0$. Hinloopen et al. (2013) were able to show the existence of the value function using geometrical arguments. For the present dynamic game, we are not able to proceed along those lines. We therefore propose a method to obtain numerical approximations of the value function. ${ }^{12}$

\section{Computation}

This section discusses the numerical solution strategy. First, a number of preliminary space and time transformations bring the Hamilton-Jacobi-Bellman equations into the form of a system of quasi-linear parabolic partial differential equations with constant and isotropic diffusion tensors with given initial values. In order to obtain numerical solutions, artificial boundary conditions have to be imposed on the problem; this is done in such a way that the solutions are not essentially changed. A standard numerical scheme, the method of lines, can then be used to obtain numerical approximations of the solutions to the resulting system.

\subsection{Preliminary transformations}

The structure of equation (20) for $V^{i}$ is that of a parabolic partial differential equation with the statedependent diffusion tensor

$$
D=\varepsilon\left(\begin{array}{cc}
c_{1}^{2} & 0 \\
0 & c_{2}^{2}
\end{array}\right) .
$$

The variable transformation

$$
c_{i}=e^{-x_{i}},
$$

converts $D$ to a constant multiple of the identity. For applying Itô's theorem shows that in $x_{i}$ coordinates the state equation (5) takes the form

$$
\mathrm{d} x_{i}=\left(\left(k_{i}+\beta k_{j}\right) \phi-1+\varepsilon\right) \mathrm{d} t-\sqrt{2 \varepsilon} \mathrm{d} B_{i}
$$

for $i, j \in\{1,2\}, i \neq j$.

Set $\tilde{V}^{i}\left(x_{1}, x_{2}\right)=V^{i}\left(e^{-x_{1}}, e^{-x_{2}}\right)$, etc. In the new variables, the Hamilton-Jacobi-Bellman equations read as

$$
\begin{aligned}
& \rho \tilde{V}^{i}-\tilde{V}_{t}^{i}=\varepsilon \tilde{V}_{x_{1} x_{1}}^{i}+ \varepsilon \tilde{V}_{x_{2} x_{2}}^{i} \\
&+\max _{k_{i} \geq 0}\left[\tilde{g}_{i}\left(x_{1}, x_{2}\right)-k_{i}^{2}+\tilde{V}_{x_{i}}^{i}\left(\left(k_{i}+\beta \tilde{\Gamma}_{j}^{*}\left(t, x_{1}, x_{2}\right)\right) \phi-1+\varepsilon\right)\right. \\
&\left.\quad+\tilde{V}_{x_{j}}^{i}\left(\left(\tilde{\Gamma}_{j}^{*}\left(t, x_{1}, x_{2}\right)+\beta k_{i}\right) \phi-1+\varepsilon\right)\right],
\end{aligned}
$$

\footnotetext{
${ }^{12}$ Kossioris et al. (2008) numerically compute a non-linear feedback Nash equilibrium for a differential game with a single state variable, limiting themselves to a class of continuous feedback rules. Dockner and Wagener (2014) study necessary conditions for feedback equilibria in games with a single state variable. Through an auxiliary system of differential equations they are also able to find non-continuous feedback strategy equilibria.
} 
with terminal condition

$$
\lim _{t \rightarrow \infty} \tilde{V}^{i}\left(t, x_{1}, x_{2}\right) e^{-\rho t}=0 .
$$

In order not to overburden notation, we shall drop the tildes, and refer to 'functions in $c_{i}$-coordinates' and 'functions in $x_{i}$-coordinates' instead.

The terminal condition, which specifies the value at infinite times, is numerically inconvenient. To circumvent it, the model is modified in two steps. First, the corresponding finite horizon model, where $t$ ranges from 0 to $T>0$, is considered instead, with value functions $V_{T}^{i}$ and terminal condition

$$
V_{T}^{i}\left(T, x_{1}, x_{2}\right)=0, \quad i=1,2 .
$$

Then, by replacing the variable $t$, denoting elapsed time, with the time-to-completion $s=T-t$, the terminal condition is transformed into an initial condition. Introduce the 'time-reversed value functions' by the relation

$$
V_{T}^{i}\left(t, x_{1}, x_{2}\right)=U_{T}^{i}\left(T-t, x_{1}, x_{2}\right), \quad i=1,2 .
$$

The terminal condition (26) is then replaced by the initial condition

$$
U_{T}^{i}\left(0, x_{1}, x_{2}\right)=0, \quad i=1,2 .
$$

As all time-reversed value functions $U_{T}^{i}$ satisfy the same Hamilton-Jacobi-Bellman equation for each $T$, and as they all have the same initial value, it follows by standard uniqueness results that they are equal. Hence, we may drop the subscript $T$. The time-reversed Hamilton-Jacobi-Bellman equation for $U^{i}$ then reads as

$$
\begin{aligned}
\rho U^{i}+U_{s}^{i}=\varepsilon U_{x_{1} x_{1}}^{i}+ & \varepsilon U_{x_{2} x_{2}}^{i} \\
+\max _{k_{i} \geq 0}[ & g_{i}\left(x_{1}, x_{2}\right)-k_{i}^{2}+U_{x_{i}}^{i}\left(\left(k_{i}+\beta \Gamma_{j}^{*}\left(s, x_{1}, x_{2}\right)\right) \phi-1+\varepsilon\right) \\
& \left.+U_{x_{j}}^{i}\left(\left(\Gamma_{j}^{*}\left(s, x_{1}, x_{2}\right)+\beta k_{i}\right) \phi-1+\varepsilon\right)\right] .
\end{aligned}
$$

A solution to this equation will yield all value functions $V_{T}^{i}$ by relation (27).

In the infinite horizon game, profit functions as well as state equations are autonomous, not depending explicitly on time. As a consequence, when the game is stopped at any point in time, the continuation game is identical to the original game - this could be termed the autonomous dynamic programming principle. Consequently, the present-time value functions are time-invariant. Moreover, if

$$
V_{T}^{i}\left(t, x_{1}, x_{2}\right) \rightarrow v^{i}\left(x_{1}, x_{2}\right) \quad \text { and } \quad \frac{\partial V_{T}^{i}}{\partial t}\left(t, x_{1}, x_{2}\right) \rightarrow 0
$$

as $T \rightarrow \infty$, then $v^{i}$ solves a stationary equation. Equivalently, the time-reversed value function must become asymptotically time-invariant: $U^{i}\left(s, x_{1}, x_{2}\right) \rightarrow u^{i}\left(x_{1}, x_{2}\right)$, with $u^{i}$ solving the stationary HamiltonJacobi-Bellman equations

$$
\begin{aligned}
\rho u^{i}=\varepsilon u_{x_{1} x_{1}}^{i}+\varepsilon & u_{x_{2} x_{2}}^{i} \\
+\max _{k_{i} \geq 0}[ & g_{i}\left(x_{1}, x_{2}\right)-k_{i}^{2}+u_{x_{i}}^{i}\left(\left(k_{i}+\beta \Gamma_{j}^{*}\left(x_{1}, x_{2}\right)\right) \phi-1+\varepsilon\right) \\
& \left.+u_{x_{j}}^{i}\left(\left(\Gamma_{j}^{*}\left(x_{1}, x_{2}\right)+\beta k_{i}\right) \phi-1+\varepsilon\right)\right] .
\end{aligned}
$$

Once the value functions $u^{i}$ have been determined from these equations, time-independent equilibrium 
feedback strategies are obtained as the maximizers of the right-hand side of Equation (29):

$$
\Gamma_{i}^{*}\left(x_{1}, x_{2}\right)=\max \left\{\frac{1}{2} \phi\left(u_{x_{i}}^{i}+\beta u_{x_{j}}^{i}\right), 0\right\} .
$$

To obtain an approximating numerical solution to the infinite horizon game, the system (28) is considered as an ordinary differential equation in the space of pairs $\left(U^{1}, U^{2}\right)$ of value functions depending on $x_{1}$ and $x_{2}$ with a given initial value. A solution to the stationary equation (29) is a steady state of this differential equation.

By integrating equation (28), or rather a discretized approximation, over time-to-completion $s$, and stopping once the time derivative $\left(U_{s}^{1}, U_{s}^{2}\right)$ is sufficiently close to zero, an approximation of an attracting steady state of the ordinary differential equation in function space is obtained. ${ }^{13}$

\subsection{Numerical Method of Lines}

The numerical method of lines (Schiesser, 1991) is used to obtain an approximation of the solutions to (28). Solutions to the differential equations are considered on a symmetric square region

$$
\Omega=(\underline{M}, \bar{M}) \times(\underline{M}, \bar{M}) .
$$

The region is discretized using a uniform tensor grid with grid spacing $h=(\bar{M}-\underline{M}) /(n+1)$.

Grid points are of the form $\left(x_{1, k}, x_{2, m}\right)$, with

$$
x_{1, k}=\underline{M}+k h, \quad x_{2, m}=\underline{M}+m h, \quad 1 \leq k, m \leq n .
$$

Function values at grid points are denoted as

$$
U_{k, m}=U\left(x_{1, k}, x_{2, m}\right), \quad 1 \leq k, m \leq n
$$

At each grid point $\left(x_{1, k}, x_{2, m}\right)$, derivatives are approximated by second-order central finite differences, e.g.,

$$
\begin{aligned}
\frac{\partial U^{i}}{\partial x_{1}}\left(s, x_{1, k}, x_{2, m}\right) & =\frac{U_{k+1, m}^{i}-U_{k-1, m}^{i}}{2 h}+O\left(h^{2}\right), \\
\frac{\partial^{2} U^{i}}{\partial x_{1}^{2}}\left(s, x_{1, k}, x_{2, m}\right) & =\frac{U_{k-1, m}^{i}-2 U_{k, m}^{i}+U_{k+1, m}^{i}}{h^{2}}+O\left(h^{2}\right), \quad \text { etc. }
\end{aligned}
$$

Discretizing in this way results in a system of $2 n^{2}$ ordinary differential equations. This system is however not closed, as the derivative discretizations at near-boundary points, e.g. for $k=1$, refer to undefined values, like $U_{0, m}^{i}$. These values are supplied by the boundary conditions of the problem, which are discussed in the next subsection.

The time variable is however still continuous. After discretization, a system of ordinary differential equations is obtained, approximating the partial differential equation (28). It is solved using a third-order

\footnotetext{
${ }^{13}$ This approach resembles what is in the literature known as a method of false transients (see Schiesser, 1991), where a time derivative which is not part of the original problem is added to a partial differential equation in order to transform it into a well-posed initial value (Cauchy) problem. It is then expected that this additional term will have an insignificant effect on the final solution. In our case, it is rather a method of true transients as $U_{s}^{i}$ (or $V_{t}^{i}$ ) is a true part of the Hamilton-Jacobi-Bellman equation (corresponding to a finite-horizon game) and approaches zero only in the limit (when the horizon of the game approaches infinity and the game itself becomes stationary).
} 
Runge-Kutta method (Judd, 1998). ${ }^{14}$

\subsubsection{Boundary conditions}

We already motivated our choice of the initial condition $U^{i}\left(0, x_{1}, x_{2}\right)=0$. To solve the system of differential equations (28), we also need to specify boundary conditions corresponding to the four sides of the grid square. The problem is that the value of a solution at all boundaries is ex ante not known. We address this in Appendix B, where we argue that the misspecification of the boundary conditions only results in a significant error in a small region along the boundaries. We used standard Neumann boundary conditions:

$$
\frac{\partial}{\partial \vec{n}} U^{i}(s, x)=0, \quad i=1,2,
$$

where for a boundary point $x \in \partial \Omega, \vec{n}$ is the outward pointing normal vector, and $\frac{\partial U}{\partial \vec{n}}=\vec{n} \cdot \nabla U$ is the normal derivative of $U$ at $x$.

\subsubsection{Equilibrium and time paths}

The equilibrium we compute is the limit equilibrium of a finite-horizon game as the horizon grows to infinity. This selection criterion is often used in the literature (see, for instance, Chen et al. (2009)). When computing the finite horizon equilibria using symmetric terminal conditions, we naturally find symmetric strategy equilibria, that is, equilibria for which in state $\left(c_{1}, c_{2}\right)=\left(c^{\prime}, c^{\prime \prime}\right)$ firm 1 exerts the same R\&D effort as firm 2 in state $\left(c^{\prime \prime}, c^{\prime}\right)$. As firms in our model face the same demand and cost primitives, this symmetry in their behaviour is guaranteed.

As a robustness check, we ran the finite horizon algorithm with homogeneous agents facing asymmetric terminal conditions. In all of these simulations, the algorithm converged to the same symmetric strategy equilibrium, fortifying our conjecture that this is the unique equilibrium for this game. ${ }^{15}$ Note however that in our symmetric strategy equilibrium, ex-post asymmetries in the cost levels between firms can and do arise endogenously as a result of firms' R\&D decisions and random shocks to production costs.

Having obtained numerical approximations of the value functions in (28) and consequently the equilibrium feedback strategies, the investment paths of firms can be simulated. Stochastic time paths are calculated using the Euler-Maruyama scheme (Kloeden and Platen, 1992). For low values of $\varepsilon$, the drift part of equation (24) generates a good approximation of the evolution of the state variables over time. That is, we solve

$$
\dot{x}_{i}=\left(\hat{\Gamma}_{i}^{*}+\beta \hat{\Gamma}_{j}^{*}\right) \phi-1+\varepsilon, \quad x_{i}(0)=x_{i}^{0}, \quad i=1,2,
$$

where $\hat{\Gamma}_{i}^{*}\left(x_{1}, x_{2}\right)$ and $\hat{\Gamma}_{j}^{*}\left(x_{1}, x_{2}\right)$ are obtained from (30) after replacing derivatives of the value functions with their numerical approximations, and where $x_{i}^{0}$ is firm $i$ 's initial value of unit cost. Any value of variables between grid points is obtained using cubic spline interpolation (Judd, 1998). Steady states of the drift vector field are analyzed in the usual way.

\footnotetext{
${ }^{14}$ We wrote the code for computations in Fortran 95, using double precision arithmetic. The criterion for convergence is that the value of the $L^{2}$-norm of $\mathbf{U}_{s}$ is below $1 \times 10^{-12}$. Auxiliary calculations and plots were executed in MATLAB and Mathematica. In presented plots, $\underline{M}=-2.5, n=200$ and $h=0.035$. To prevent the solution from becoming unstable, the time step $\Delta t$ in the Runge-Kutta method has to be taken sufficiently small, in order to satisfy both the Courant-Friedrichs-Lewy condition $\Delta t<\frac{h}{v}$, where $v$ is a maximum drift velocity, and the diffusion condition $\Delta t<\frac{h^{2}}{2 \varepsilon}$. For small $\varepsilon$, as used in the article, the latter condition is usually not binding.

${ }^{15}$ Indeed, many comparable papers in the field of dynamic games even a priori decide to compute only symmetric Markov perfect equilibria, e.g., Chen et al. (2009), Besanko et al. (2010).
} 


\section{Equilibrium strategies and industry dynamics}

This section presents the results of the numerical analysis. It discusses strategic interactions between firms as implied by their policy (R\&D) functions. Furthermore, state vector fields and time paths of certain variables of interest are analyzed to obtain insight into possible evolutions of the game.

The nature of equilibrium dynamics primarily depends on the relationship between profit potential $(\phi)$ and discount rate $(\rho) .{ }^{16}$ From an exhaustive exploration of the parameter space, we identify three qualitatively distinct stable types of dynamics - stable in the sense that a sufficiently small change in parameter values does not lead to a qualitative change of the dynamics. The first one is Promising Technology (high $\phi$ for a given $\rho$ ). In this case, which will be the center of our attention, firms find it profitable to develop further a technology which requires $R \& D$ efforts before the production can profitably start. Indeed, for great many new technologies, research starts long before a prototype sees the light of day. When $\phi$ is moderate for a given $\rho$, we obtain the second type of dynamics, which we call a Strained Market. In this case, only technologies allowing for immediate production are developed further and even those only if they are already sufficiently developed, such that they do not require 'too much' additional $\mathrm{R} \& \mathrm{D}$ efforts. When $\phi$ is relatively small for a given $\rho$, we obtain the third type of dynamics, which we call Obsolete Technology. In this case, it is always in the interest of any firm to exit the market at some optimal speed as low market revenues make it unprofitable to maintain a decaying technology.

In what follows, we discuss the three types of dynamics one by one and consider how different levels of spillovers affect each. We present those findings that appear robust throughout the parameter space as Results. While plots are drawn in original state variables, we usually preserve the logarithmic scale for the sake of clarity.

\subsection{Equilibrium Dynamics I: Promising Technology}

Figure 2 shows the value function and R\&D efforts for an intermediate level of spillovers $(\beta=0.5)$. As the value functions are symmetric in the sense that $V^{2}\left(c_{2}, c_{1}\right)=V^{1}\left(c_{1}, c_{2}\right)$, it is sufficient to consider just $V^{2}$, which is shown in plot (a) in Figure 2. Plots (b) and (c) in Figure 2 show the symmetry between policy functions. Observe that a firm's value function is negatively related to a firm's own unit cost and positively related to the unit cost of its competitor. The smaller a firm's unit costs for a given cost of its competitor, the better a firm's competitive position and so the larger the profits a firm is able to reap. The highest, left part of the firm 2's value function corresponds to unit costs for which firm 2 is a monopolist. Firm 2's relative cost advantage keeps its competitor out of the market. For lower values of firm 1's unit costs, both firms are (eventually) active in the market (recall Figure 1). This change of the regimes is marked by a steep decline in the value function of the incumbent firm. For relatively high values of own unit costs (the bottom part), the value of the game for firm 2 is zero as the firm finds it optimal to stay inactive; the technology is too expensive to develop.

The profits a firm is able to reap from the product market are determined by a firm's cost efficiency. The latter is costly in the sense that due to a positive rate of technology depreciation, a firm needs to invest in R\&D not only to increase its efficiency (relative to its competitor), but also to maintain it. Observe in Figure 2 that, for a given unit cost of its competitor, the $R \& D$ effort of a firm increases with decreasing own unit cost over the region of zero production and decreases shortly thereafter. This is driven by the pure cost effect, which positively affects a firm's incentives for R\&D. When initial unit costs are high (but still low enough for a firm to pursue further development), there are huge benefits from exerting

\footnotetext{
${ }^{16}$ Recall from Lemma 1 that $\rho=r / \delta$, such that a higher $\rho$ is either due to a higher discount rate $(r)$, or a lower depreciation rate $(\delta)$, in which case cost reductions take longer.
} 


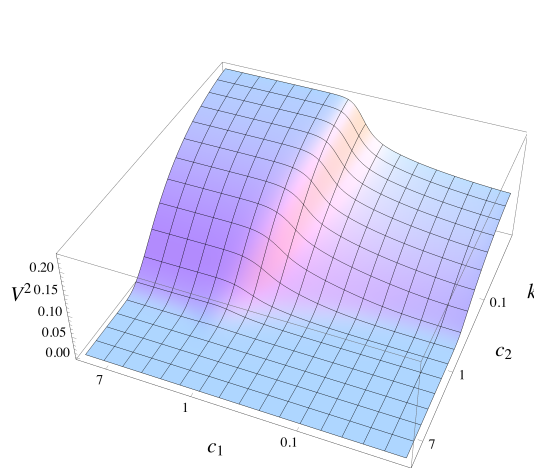

(a)

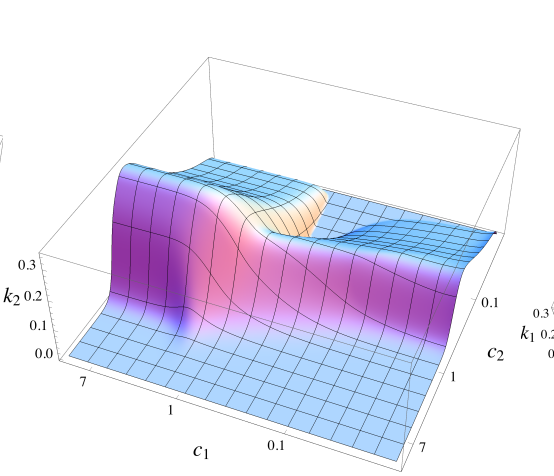

(b)

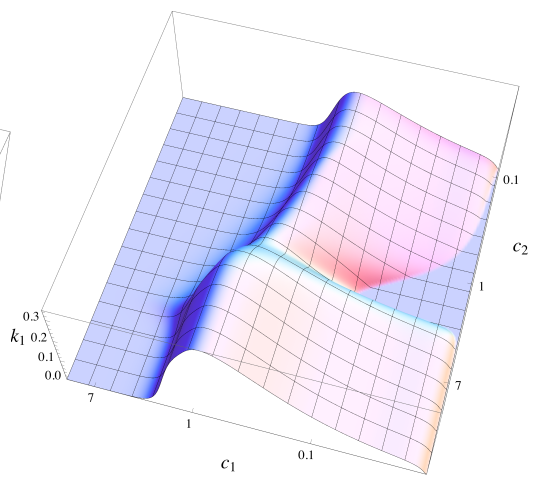

(c)

Figure 2: Firm 2's value function (a) and R\&D efforts (b), together with Firm 1's R\&D efforts (c). Parameters: $(\phi, \rho, \beta, \varepsilon)=(8,1,0.5,0.125)$.

R\&D efforts as this reduces the amount of time needed to reach the production phase. Consequently, $R \& D$ efforts are high. However, any R\&D effort a firm exerts contributes also to the reduction of a competitor's production costs, which retroactively negatively affects the firm's profits in the product market competition. This feedback cost effect, which is greater the higher the spillovers, diminishes the incentives of a firm for R\&D.

\subsubsection{The evolution of technology and market structure}

Recall from Figure 1 that there are three possibilities in the product market, depending on the value of unit costs: no production at all, duopoly, or monopoly by one of the two firms. They are delimited by the the product market "entry/exit" curves of firm 1 and firm 2 ( $E_{1}$ and $E_{2}$, respectively). For any starting value of unit costs, we are interested in how the spillovers affect the way in which firms steer their unit costs as the game evolves. We first consider the dynamics at medium spillovers $(\beta=0.5)$ and afterwards compare it to the dynamics at low and high spillovers. There are many possibilities for the industry dynamics. For instance, high initial unit costs can lead to no market (if both firms refrain from development), to monopoly (if only one firm pursues development), or to duopoly (if both firms enter the market either simultaneously or sequentially). Monopoly by one firm can either sustain or transform into duopoly if the laggard firm catches up. Likewise, duopoly can persist or change into monopoly if one firm is squeezed out of the market by the other, more efficient firm.

The R\&D efforts that both firms exert influence the way in which unit costs evolve over time through the drift term (see equation (15)). This evolvement of costs as governed by the drift term is summarized by the drift vector field in the left plot of Figure 3. This field displays velocity vectors for production costs as arrows with components $\left(\dot{c}_{1}, \dot{c}_{2}\right)$ at grid points $\left(c_{1}, c_{2}\right)$, where longer arrows indicate faster movement. ${ }^{17}$

Solutions $c_{i}^{\varepsilon}(t)$ to the stochastic evolution equation (15) tend, for finite times, to the corresponding solution $c_{i}^{0}(t)$ to the deterministic equation as $\varepsilon \rightarrow 0$ (Freidlin and Wentzell, 1998). In the following, drift paths are used as an approximation of the evolution of the system over time.

In Figure 3, the $\dot{c}_{1}=0$ loci (labeled $I_{1}$ ) and $\dot{c}_{2}=0$ loci (labeled $I_{2}$ ) intersect in four steady states of the drift vector field: $S_{1}$ and $S_{2}$ are saddles, $S_{3}$ is a nodal source, whereas $S_{4}$ is a nodal sink. Invariant manifolds of the two saddles are labeled by letter $W$. Stable and unstable manifolds of $S_{1}$ are labeled by $W_{1}^{S}$ and $W_{1}^{U}$, respectively. Similarly, $W_{2}^{S}$ and $W_{2}^{U}$ are, respectively, a stable and an unstable manifold

\footnotetext{
${ }^{17}$ Vectors $\left(\dot{c}_{1}, \dot{c}_{2}\right)$ are analogous to vectors $\left(\dot{x}_{1}, \dot{x}_{2}\right)$, as defined in (32), and are scaled so that arrows do not overlap.
} 

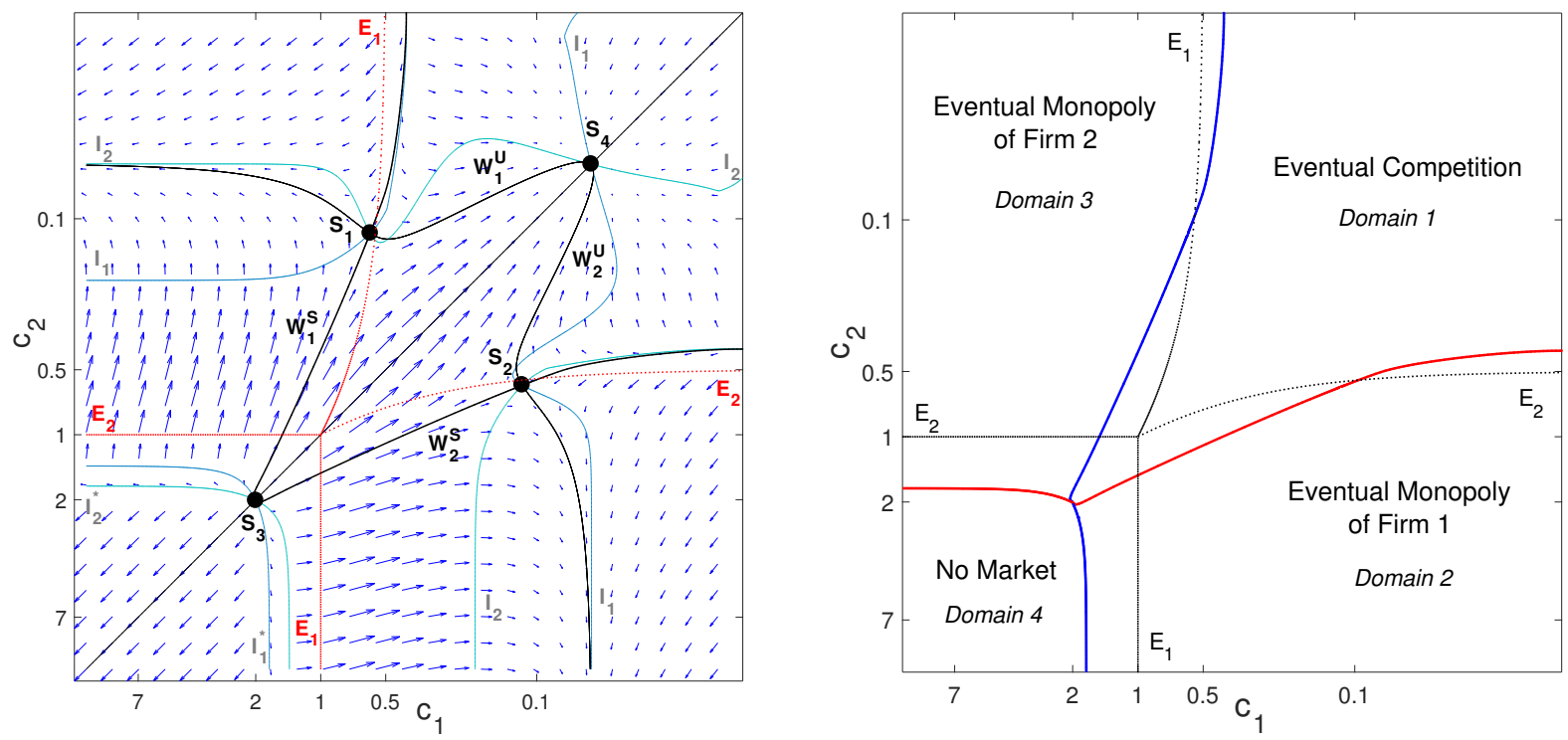

Figure 3: Drift vector field for $\beta=0.5$ (left) and its domains (right), $(\phi, \rho, \varepsilon)=(8,1,0.125)$.

of $S_{2} .{ }^{18} E_{1}$ and $E_{2}$ are the product-market "entry/exit" curves of firm 1 and firm 2, respectively. In the region above the indicated 45-degree diagonal, firm 2 has a cost advantage over firm 1, whereas the reverse is true in the region below the diagonal. For combinations of unit costs lying exactly on the diagonal, the firms are equally efficient. Observe that the vector field below the indicated 45-degree diagonal is a mirror image of the field above the diagonal. This follows from the symmetry of the feedback equilibrium, best visible in the last two plots of Figure 2.

Notice that $W_{1}^{S}$ and $W_{2}^{S}$ are separatrices which together with isoclines $I_{1}^{*}$ and $I_{2}^{*}$ around $S_{3}$ divide the state space into four domains (outlined in the right plot of Figure 3). The first domain is a basin of attraction of the asymptotically stable steady state $S_{4}$. Every motion starting in this domain converges to $S_{4}$ as $t \rightarrow \infty$. In this domain, eventually both firms are active in the product market. In any other domain, the unit cost of at least one firm diverges to infinity; we are left either with a monopoly of firm 1 (domain 2), a monopoly of firm 2 (domain 3), or no market at all (domain 4). Observe that the limits of these domains do not coincide with the product-market "entry/exit" curves.

We analyze possible evolutions of the game by jointly looking at Figure 2 and Figure 3. In the bottom-left corner, the unit costs of both firms are "very" high and above the choke price, such that both firms decide to refrain from developing further the initial technology. Future expected profits are not high enough to compensate for investments needed to bring the technology to the production phase. Technically, unit costs flow towards infinity due to the positive depreciation rate. Intuitively, though, we always interpret any situation in which a firm stays inactive as one in which it has left the market.

Left to the stable manifold of $S_{1}$, labeled in Figure 3 by $W_{1}^{S}$, the cost advantage of firm 2 over firm 1 is so large that the latter gives up on R\&D (see figure 2). When cost asymmetries are large, the profits the less efficient firm earns in the product market are low. This reduces the ability of firm 1 to compensate for R\&D investments needed to bring its technology to the product market and catch up with firm 2. It turns out that left to $W_{1}^{S}$ the cost asymmetries are just so large that firm 1 cannot even afford to battle depreciation of its own technology, thereby succumbing to its more efficient competitor. Firm 1 does produce only when its initial unit costs are already sufficiently low (the region between $E_{1}$ and $W_{1}^{S}$

\footnotetext{
${ }^{18}$ The stable and the unstable manifold are the only two trajectories that pass through the saddle point. On any trajectory other than the stable path, the direction of motion is always away from the saddle point.
} 
curve at the very top), such that it can profitably sell a positive quantity in a competitive product market. However, its product market activity is only temporary as the large cost advantage enables the more efficient firm 2 to squeeze firm 1 out of the market. Thus, firm 1 does eventually neither produce nor invest in R\&D. Its unit costs in this region always tend to flow towards infinity, which is, again, due to the positive depreciation rate.

The investment of firm 2 depends on its initial unit cost. The firm decides to enter the market for all initial costs that in Figure 3 correspond to $c_{2}$ above the $I_{2}^{*}$ curve which flows through $S_{3}$. For initial unit costs above the choke price ( $c_{2}$ below the horizontal part of $E_{2}$ curve), the firm does at first produce nothing but invests increasingly in the reduction of its unit costs. Once its unit costs have been reduced below the choke price, the firm starts producing as it can now sell at positive mark-ups. The level of $\mathrm{R} \& \mathrm{D}$ efforts and unit costs then gradually decrease to their long-run optimal levels ( $c_{2}$ approaches the unstable manifold $W_{1}^{U}$ which asymptotically converges with $I_{2}$ isocline). For unit costs above the choke price, instantaneous profits of firm 2 are negative as there is no production yet. Firm 2 initiates R\&D as it expects future profits will more than compensate for initial investments. That is, while initially instantaneous profits are negative, the expected total discounted profits are positive. There exists a finite upper bound on unit costs beyond which expected future profits are not enough to compensate for short run losses (unit costs corresponding to $c_{2}$ below the $I_{2}^{*}$ curve). In this case, the initial technology is not developed further. Observe how the direction of vectors in Figure 3 changes its sign when passing through the $I_{2}^{*}$ curve.

Below the $W_{2}^{S}$ curve, the situation is reversed: now the cost advantage of firm 1 brings about a monopoly. For all initial unit costs on the right side of the $I_{1}^{*}$ curve passing through $S_{3}$, firm 1 brings a technology into the market, while firm 2 is sooner or later forced out of business.

In the upper-right part of the state space, between the $W_{1}^{S}$ and $W_{2}^{S}$ manifolds, the cost asymmetries are moderate. Eventually, a product market duopoly emerges as for all initial costs in this region, each firm sooner or later brings a technology into the product market. It is interesting to observe that the asymptotically stable steady state $S_{4}$ lies on the 45-degree diagonal. This implies a kind of a regression toward the mean phenomenon, where any initial difference in the unit costs between firms tends to vanish over time. ${ }^{19}$ We have noted that above the 45 -degree diagonal, firm 2 has a cost advantage, which is to the left of $W_{1}^{S}$ large enough to squeeze firm 2 out of the market. However, to the right of $W_{1}^{S}$, this is not the case any more. Notice that $W_{1}^{S}$ curve travels along the edge of the precipice in the policy function of firm 1 (see the right-most plot in Figure 2). While left to $W_{1}^{S}$ firm 1 gives up on R\&D, right to $W_{1}^{S}$, it invests heavily to catch up with firm 2 . Firm 2 exerts less R\&D efforts than firm 1, however, it prolongs its cost supremacy through positive spillover effects arising from relatively high $R \& D$ efforts of firm 1 . When its own initial costs are very low, firm 2 for some time even sits back on R\&D (observe a basin in the upper part of the firm 2's policy function in the mid plot of Figure 2) and retards its technology decay optimally by relying mostly on spillovers from the R\&D efforts of its zealous counterpart. ${ }^{20}$ Namely, when unit costs of firm 2 decrease relative to firm 1, an additional unit of firm 2's R\&D effort benefits firm 1 progressively more than firm 2 itself, which diminishes firm 2's incentives for own R\&D (this follows directly from the formulation of unit costs in (2)). The story is analogous when we are on the other side of the diagonal, where firm 1 has a relative cost advantage. In both cases, a dominant firm gradually loses its lead.

\footnotetext{
${ }^{19}$ We say "tends to" as by considering the drift vector field we are disregarding random shocks. In a stochastic game, only the gap between the mean values of the two unit costs narrows and eventually closes.

${ }^{20} \mathrm{~A}$ typical example of a large firm relying on inventions by smaller firms is supposedly Microsoft, whose competitors "have long complained that the rest of the industry has served as Microsoft's R\&D lab" (Pollack, 1991).
} 


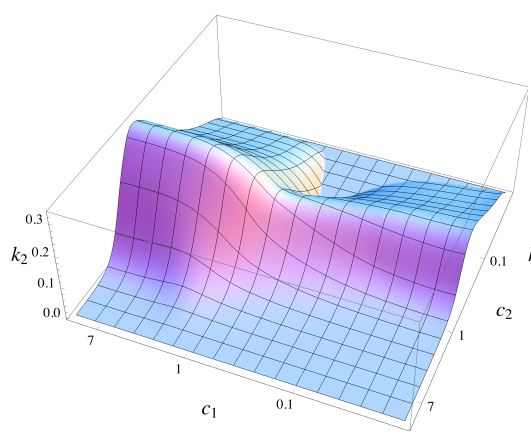

(a) $\varepsilon=0.25$

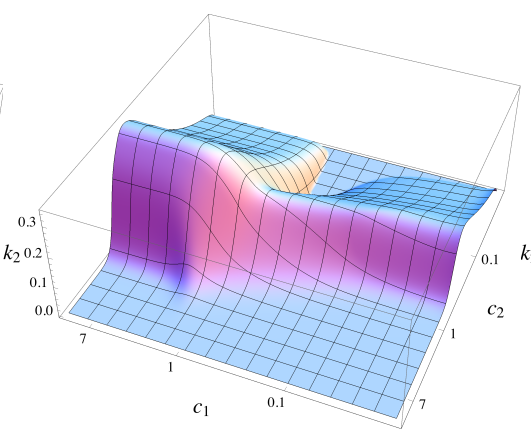

(b) $\varepsilon=0.125$

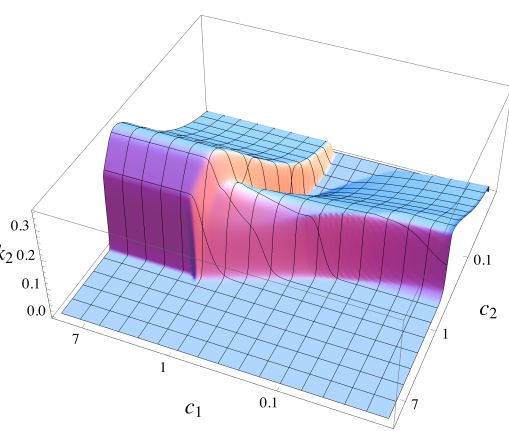

(c) $\varepsilon=0.0156$

Figure 4: Firm 2's policy function for different levels of uncertainty, $(\phi, \rho, \beta)=(8,1,0.5)$.

\subsubsection{Stochasticity and R\&D}

In this section we consider how R\&D efforts of firms relate to uncertainty. Figure 4 shows that the policy function is smoother for higher levels of noise $(\varepsilon)$ in unit costs. ${ }^{21}$

To investigate this further, we plot the value function and policy function of firm 2 for different fixed values of firm 1's unit cost. In Figure 5, we fix $c_{1}$ at such a high value that firm 2 is a monopolist ( $c_{1}=11.76$; for reference, $A=1$ ). Then, we can directly compare our solution with the deterministic monopoly solution, obtained in Hinloopen et al. (2013). The deterministic value function (the solid curve) has a kink at an indifference point, which is the value of the unit cost for which the firm is indifferent between developing the technology further and exiting the market. In contrast, the stochastic value function is smooth. The fact that the value function corresponding to a higher noise level lies above the one corresponding to a lower noise level suggests that stochasticity increases expected profits. ${ }^{22}$

We see that the stochastic value function converges to the deterministic monopoly value function as $\varepsilon \rightarrow 0$. For $\varepsilon=0.0156$, the stochastic solution is already almost indistinguishable from the deterministic one, the absolute difference between the two solutions at the kink being 0.0012 . The deterministic policy function is discontinuous at the indifference point. This discontinuity is smoothed out by stochasticity. The policy function of the stochastic model is smooth and everywhere differentiable. It is interesting to observe that stochasticity makes a firm invest in R\&D over the values of unit costs for which a firm in the deterministic setting already gives up. The firm in the stochastic setting still invests a bit at larger costs in hope of a favorable shock, for which it sacrifices some investments at lower unit costs - the R\&D efforts are smoothed out. ${ }^{23}$ While R\&D efforts exerted at large costs might as such not be sufficient to bring a technology to the production phase, they at least retard the decay of a technology for some time during which hopefully a favorable shock arises. Due to the depreciation of its technology, the firm gradually gives up on $R \& D$ if no favorable shock of a sufficient size occurs, but more slowly so the larger the variance of shocks. The higher uncertainty, therefore, leads to more opportunistic behavior of

\footnotetext{
${ }^{21}$ To get some idea about the uncertainty implied by different values of $\varepsilon$, suppose that firms invest in R\&D just enough to offset the depreciation of technology. That is, suppose that the drift in the state equation (5) is zero $(\mu=0)$, and so the movement of $c_{i}$ is driven purely by random stochastic shocks. Further, observe from (5) that $c_{i}(t)$ is a log-normally distributed random variable which by definition takes only positive real values. With a zero drift, this means that $\ln c_{i}(t) \sim \mathcal{N}\left(\ln c_{i}(0)-\varepsilon T, 2 \varepsilon T\right)$. The $100(1-\alpha) \%$ confidence interval for $c_{i}$ is then $\exp \left(\ln c_{i}(0)-\varepsilon T \pm z_{\alpha / 2} \sqrt{2 \varepsilon T}\right)$. Suppose $c_{i}(0)=0.4$. Then, the $90 \%$ confidence interval for $c_{i}$ after 1 unit of time $(T=1)$ is approximately $[0.16,0.80]$ for $\varepsilon=0.125$ and $[0.29,0.53]$ for $\varepsilon=0.0156$.

${ }^{22}$ Observe from (12)-(13) that the profit function is convex in the unit cost $c$. The result then follows from Jensen's inequality (see Dixit and Pindyck (2012)), according to which greater uncertainty will increase the expected value of an action if the payoff is convex in the random variable (and decrease it if the payoff is concave).

${ }^{23}$ Clearly, when there is no uncertainty involved, a firm has no reason to initiate investment if it never enters the product market as that would mean that a firm incur costs with no future compensation for them.
} 


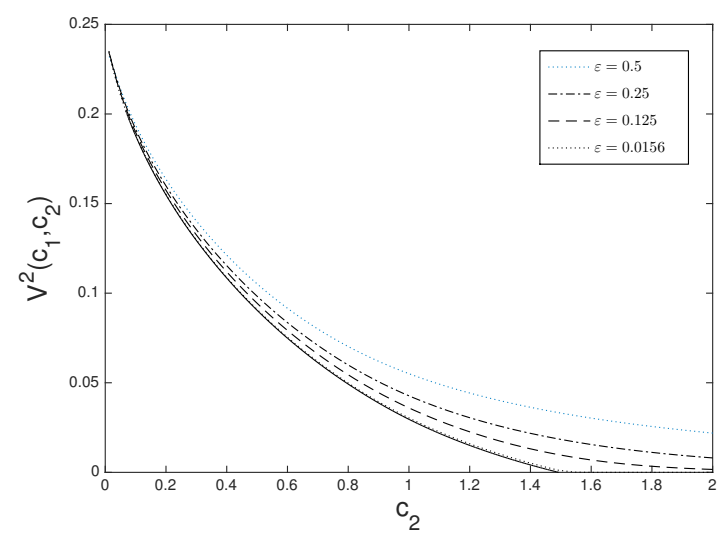

(a)

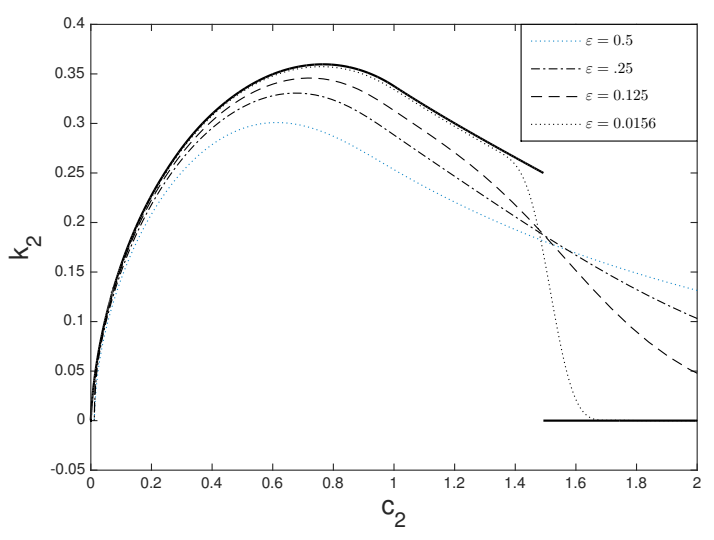

(b)

Figure 5: Value functions (a) and policy functions (b) of firm 2 for varying levels of noise $\varepsilon$ when the unit cost of firm 1 is fixed at $c_{1}=11.76$. The solid line corresponds to the deterministic monopoly solution. Parameters: $(\phi, \rho, \beta)=(8,1,0.5)$.

firms, which increases the chance that the development of expensive technologies will be pursued further. Computations shows that this opportunistic behavior also increases the relative size of the region of the state space for which eventually duopoly is likely to appear in the product market (in the drift vector field, Domain 1 spreads out with an increasing level of $\varepsilon$ ).

Result 1 (Uncertainty). The uncertainty in costs increases the likelihood that a technology will be developed to the production stage and that the resulting market will be competitive.

\subsubsection{Spillover effects}

In this section, we compare the case with medium spillovers to that of low and high spillovers. The higher the level of spillovers, the more the R\&D efforts that a firm exerts benefit its competitor, and thus the larger the role of feedback cost effects in shaping a firm's policy function. Figure 6 shows a policy function of firm 2 for low and high spillovers, whereas Figure 7 shows the corresponding drift vector fields.

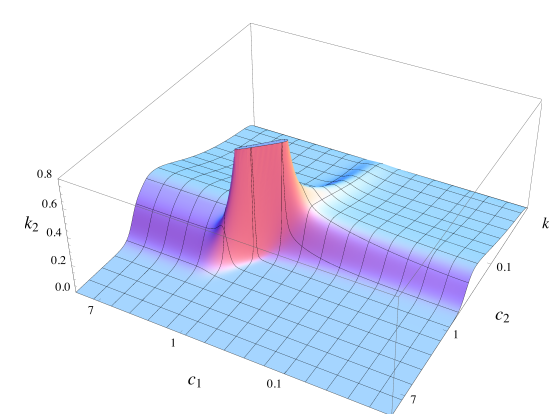

(a) $\beta=0$ (scale preserving)

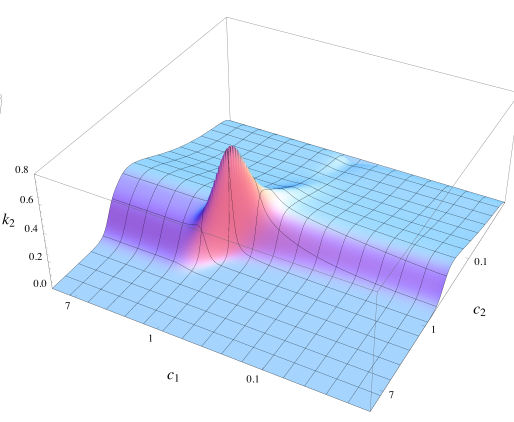

(b) $\beta=0.1$

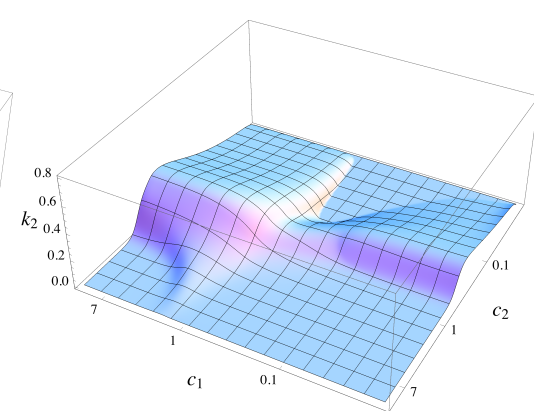

(c) $\beta=0.9$

Figure 6: Policy function of firm 2 for low and high spillovers, $(\phi, \rho, \varepsilon)=(8,1,0.125)$.

Low Spillovers. For low levels of spillovers, the policy function exhibits a sharp and narrow spike. This spike reaches higher, the lower the spillovers and the higher the value of $\phi$ for a given $\rho$. Observe in the drift vector field for $\beta=0$ (Figure 7a) that $W_{1}^{S}$ and $W_{2}^{S}$ separatrices are in the vicinity of $S_{3}$ 


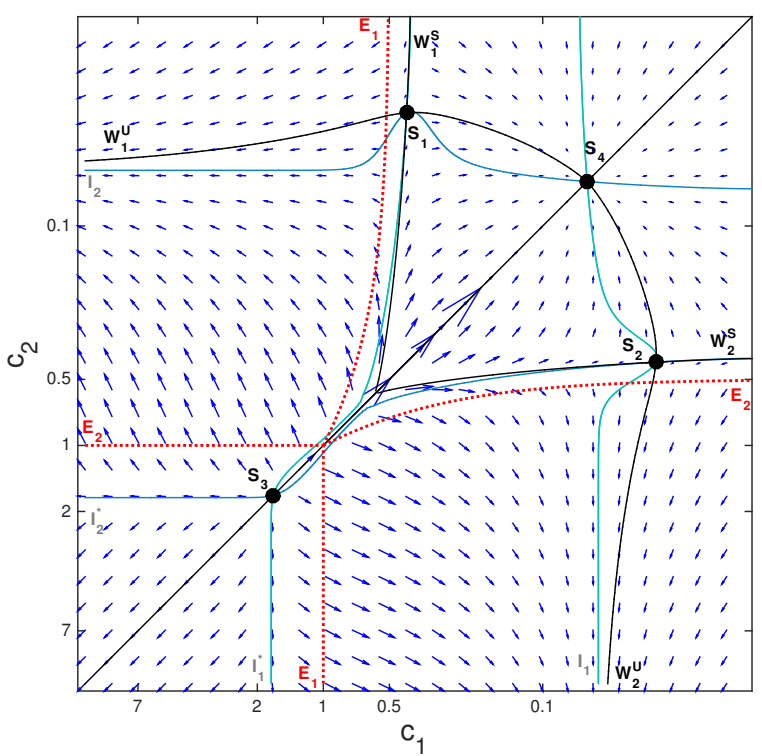

(a) $\beta=0$

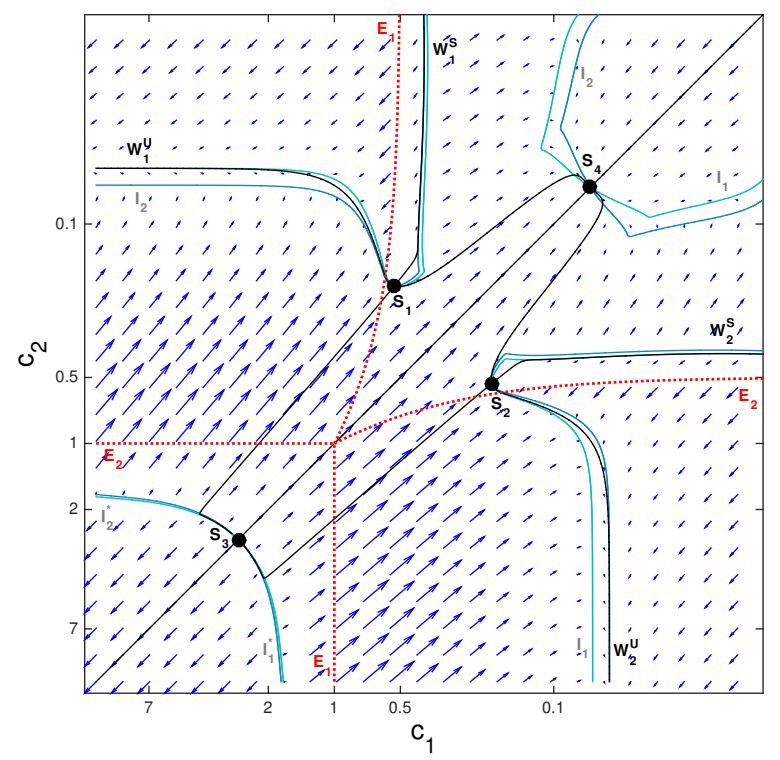

(b) $\beta=0.9$

Figure 7: Drift vector field for low spillovers (a) and high spillovers (b), $(\phi, \rho, \varepsilon)=(8,1,0.125)$.

practically indistinguishable from the diagonal - a small difference in unit costs is enough to drive the less efficient firm out of the market. This region of proximity corresponds to the location of the spike. With increasing levels of spillovers and/or noise, the spike becomes thicker and lower; the separatrices shift away from the diagonal, implying that a larger cost advantage is needed to drive the opponent out of the market (compare with Figure 3).

As Figure 8 shows, on the diagonal within the region of proximity, each firm invests a lot trying to reduce its production costs as fast as possible and so increase its chances of survival (the vertical line in the figure corresponds to symmetric costs). Observe in the first plot of Figure 7 that the region of proximity also extends below the product-market 'entry/exit' curves, such that these high investments can take place well before any production. For a symmetric initial position, firms are thus engaged in a preemption race where the one that falls sufficiently behind the other is driven out of the market.

Observe that the spike attains its peak above the diagonal (left to the vertical line in Figure 8) and sweeps sharply down on the other side of the diagonal (the policy function has a steep slope on the right side of the vertical line). The firm with a cost advantage therefore invests heavily (but briefly), whereas the laggard is induced to give up. This additional R\&D effort of the leader can be considered predatory in the sense that it is profitable only for its effect on the exit decision of the laggard, but unprofitable otherwise. ${ }^{24}$ The predatory nature of these investments is confirmed by the fact that such large investment asymmetries never occur when the likelihood that a rival remains viable is negligible (e.g., at very high levels of a rival's unit cost) or the ability of a firm to influence this likelihood is negligible (e.g., in the case of large spillovers where large investments would to a great extent benefit the competitor).

The extent of predatory efforts (the size of the spike) is positively related to the ease with which the leader can induce the laggard to give up. Recall that the spike diminishes in size when spillovers and uncertainty get larger. At low spillovers, it is easier for the leader to induce the laggard to exit as the latter

\footnotetext{
${ }^{24}$ In declaring an action predatory, we follow Cabral and Riordan (1997) who define an action as predatory if "i) a different action would increase the likelihood that rivals remain viable, and ii) the different action would be more profitable under the counterfactual hypothesis that the rival's viability were unaffected" (p. 160). Our interpretation is similar to that of Borkovsky et al. (2012) who consider predatory investment in a dynamic quality ladder model.
} 


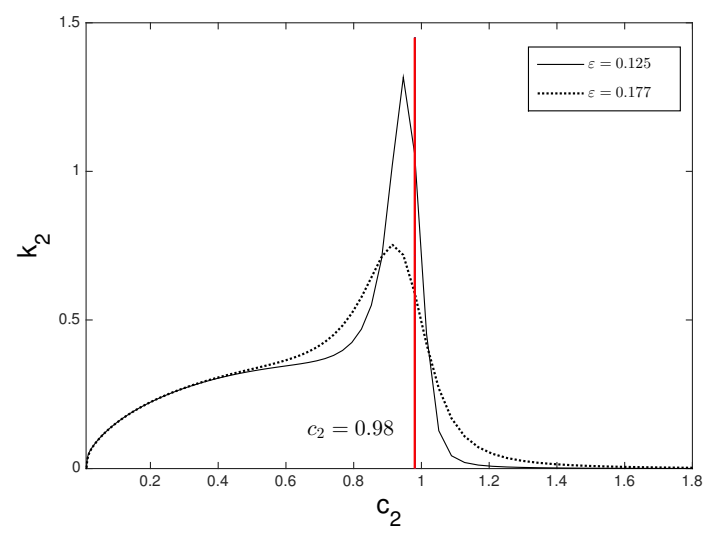

Figure 8: Policy function of firm 2 for the fixed value of firm 1's unit cost, $c_{1}=0.98$, corresponding to the region with a spike, $(\phi, \rho, \beta, \varepsilon)=(8,1,0,0.125)$.

cannot count on catching up with the leader by copying the results of the leader's R\&D efforts. Thus, the lower the spillovers, the easier it is for the leader to achieve his dominance by exerting R\&D efforts and so the higher are his incentives for extensive predation. Next, when the probability of large unexpected changes in costs is high, the laggard does not give up that fast when falling behind as it is still possible for him to catch up with the leader if he has a run of luck. In this case, the leader needs to achieve a relatively large cost advantage to induce the laggard to give up. However, due to large randomness in costs, the effect of the leader's R\&D efforts on the likelihood of achieving such an advantage is small. Consequently, his incentives for predatory investments are low. On the contrary, when there is low uncertainty in cost movements, a small cost advantage is sufficient to drive the other firm out of the market and the effect of the leader's R\&D efforts on the likelihood of achieving a needed cost advantage is large. As a consequence, the leader's incentives to engage in extensive predation are high.

Result 2 (Preemption and predation). When spillovers are low, the profit potential high, and the discount rate and uncertainty relatively low, the equilibrium is characterized by large $R \& D$ investments before production and tipping towards market monopolization when one firm gains an advantage in terms of its production cost.

High Spillovers. Figure $6 \mathrm{c}$ and Figure $7 \mathrm{~b}$ show the policy function and the drift vector field, respectively, for $\beta=0.9$. Figure 9 jointly plots the limits of market domains (cf. Figure 3) for $\beta=0$ (L), $\beta=0.5(\mathrm{M})$, and $\beta=0.9(\mathrm{H})$.

When the level of spillovers is high, the R\&D efforts by one firm benefit the other firm to a large extent. As each firm tries to free-ride on the other firm's R\&D efforts, the incentives for R\&D can be rather low. This standard conclusion in the literature is in part confirmed by our calculations $-\mathrm{R} \& D$ efforts decrease over the bulk of state space as the level of spillovers approach one. However, there are two important exception, visible in Figure 6c. First, the policy function for high spillovers extends farther into the high cost region. The intuition for this is the following. Exerting R\&D efforts is costly (recall the quadratic R\&D cost function). When spillovers are high and so R\&D efforts of the firms complement each other well, firms facing a convex cost function are able to circumvent diseconomies of scale in $R \& D$ to a large extent. Hence, the higher the spillovers, the larger the savings in $R \& D$ costs. This makes it more likely that the firms will find if profitable to develop further some initial technology. On the other hand, higher spillovers make it less likely that a firm will be able to gain a dominant position, thereby reducing its expected future mark-ups. Which effect is stronger depends on parameters, such that the relative position of $S_{3}$ for different spillovers can be different than in Figure 9 (which typically happens for lower 


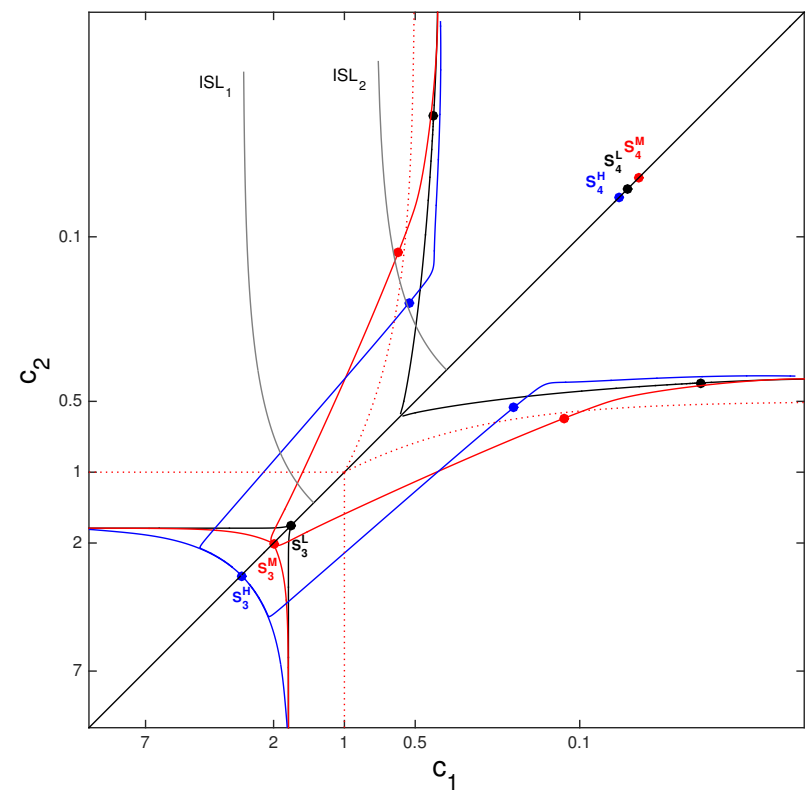

Figure 9: Comparison of market domains (separatrices of the drift vector field) between different levels of spillovers: $\beta=0$ (black), $\beta=0.5$ (red), and $\beta=0.9$ (blue); $(\phi, \rho, \varepsilon)=(8,1,0.125)$.

values of $\rho$ ). More robust is the observation that the width of the arc that separatrices $W_{1}^{S}$ and $W_{2}^{S}$ form around $S_{3}$ increases with spillovers. Therefore, when initial costs are high and firms need to invest in R\&D before production, the likelihood that the ensuing product market will be competitive increases with spillovers. This second exception at high spillovers is associated with a pronounced bulge in the policy function, spreading into the bottom part of the state space (i.e., $c_{1} \approx 1.5, c_{2}$ high in Figure $6 \mathrm{c}$ ). The size of this bulge increases with spillovers (first traces of it appear in the policy function for $\beta=0.5$ in Figure 2). The intuition is the following. In the corresponding region below the diagonal, firm 1 has a substantial cost advantage. When spillover are low, firm 2 cannot benefit much from firm 1's R\&D, such that it gives up. However, when spillovers are high, firm 2 can benefit a lot from firm 1's R\&D, which improves its prospects for catching up and thereby increases its own incentives for R\&D.

Comparing the drift vector field for $\beta=0$ to that for $\beta=0.5$ in Figure 9, we observe that Domain 1 (the basin of attraction of the steady state $S_{4}$ ) is wider in the latter case. The $W_{1}^{S}$ and $W_{2}^{S}$ separatrices spread out. This suggests that it takes a larger cost asymmetry for the less efficient firm to leave the market when spillovers are higher. In particular, the exit of any firm is much less likely when both firms already produce (for $\beta=0.5$, larger parts of separatrices lie outside the production region bounded by $E_{1}$ and $E_{2}$ curves). The higher the spillovers, the more the laggard can benefit from the R\&D investments of the leader and so the more disadvantaged it must be to give up. This point was already raised by Petit and Tolwinski $(1999$, p. 204) claiming that "[...] for a duopoly consisting of unequal competitors free diffusion of knowledge may be a way to avoid market concentration."

In what follows, we show that the pro-competitive benefit of higher spillovers does not hold for all levels of spillovers and costs. Observe how in Figure 9 the separatrices corresponding to $\beta=0.9$ (blue) intersect those corresponding to $\beta=0.5$ (red). While for high initial unit costs of firms higher spillovers still make duopoly in the ensuing product market more likely, this does not hold for lower values of initial unit costs as there the less efficient firm is sooner squeezed out of the market when spillovers are higher. Behind this result are two countervailing effects of spillovers. The first effect is a pure spillover effect the higher the spillovers, the more the laggard is able to free-ride on the leader's R\&D efforts and so the 
easier it is for him to overcome any initial asymmetries. This effect is positively related to the level of spillovers and contributes to widening the region of eventual product market duopoly. The second effect is the feedback cost effect, which is also positively related to the level of spillovers, however, it contributes to narrowing the region of eventual product market duopoly. When the unit cost of a firm is large, an additional unit of R\&D effort benefits this firm a lot (the pure cost effect dominates). However, when the unit cost of the firm is lower, so is the impact of an additional unit of R\&D on its costs (the factor $c_{i} k_{i}$ in (15) decreases with $c_{i}$ for a given $k_{i}$ ). If the unit cost of the laggard is sufficiently larger, it can well happen that the additional R\&D effort of the leader benefits the laggard more than the leader himself $\left(c_{i} k_{i}<c_{j} \beta k_{i}\right)$. As lower costs of the laggard through fiercer product market competition negatively affect the leader's profits, this reduces the leader's incentives to invest in R\&D. This feedback effect, which negatively affects the leader's R\&D efforts, is stronger, the higher the spillovers. Consequently, the higher the spillovers, the less asymmetry in costs it takes for the leader to optimally stop his R\&D efforts. Observe how the region of zero R\&D efforts above the diagonal spreads out in the policy function as spillovers increase (compare plots in Figure 4 and Figure 6). This explains why higher spillovers might increase the likelihood of market dominance. After a certain level, further increases in spillovers decrease the leader's incentives to invest rather significantly, which makes it harder for the laggard to catch up with the leader. The laggard's possibilities to copy the leader's R\&D results do increase further with increasing spillovers, however, the problem is that there is now very little or nothing to copy. In the region between the intersecting separatrices, in the upper-part of the state space in Figure 9, the leader in case of $\beta=0.9$ invests relatively less than in case of $\beta=0.5$ and this effect of lower investments by the leader dominates the pure spillover effect. Consequently, while for high spillovers, the laggard is driven out of the market, for lower spillovers, he continues to catch up with the leader. While the level of spillovers for which Domain 1 is the widest at low costs depends on parameters, the fact that it starts shrinking after a certain level of spillovers appears robust throughout parameter space.

Result 3 (Market structure). When initial production costs are high, such that firms need to invest in $R \& D$ before production, the likelihood of a competitive product market increases with spillovers. This is not necessarily the case at later stages of technological development when production costs are relatively low. Increases in spillovers are therefore not universally pro-competitive.

The long-run equilibrium level of unit costs in the case of duopoly $\left(S_{4}\right)$ depends on the level of spillovers. In Figure $9, S_{4}^{H}>S_{4}^{L}>S_{4}^{M}$. That is, the long-run unit costs are the lowest for $\beta=0.5$, the second lowest for $\beta=0$, and the highest for $\beta=0.9$. However, the ranking depends on particular parameter configurations (for some $S_{4}^{L}<S_{4}^{M}$ ). More robust is the observation that high spillovers always lead to the highest long-run costs, which is a consequence of a significant free-riding problem that pervades industries with high spillovers. This suggests that increases in spillovers decrease long-run equilibrium costs only up to a certain level, beyond which further increases in spillovers start to increase the long-run costs.

In sum, we have seen that there are two dimensions through which spillovers affect markets: first, by affecting the formation and structure of markets (likelihood of a technology being brought to production and likelihood of market dominance), and second, by affecting investments in the formed markets (longrung equilibrium costs). Our analysis shows that these effects can be of opposite sign. For instance, for parameters in Figure 9, high spillovers make it most likely that some technology will be developed at all and that the ensuing product market will (at least initially) be competitive. However, at the same time, they lead to the least developed technology (the highest production costs) in the long run.

Result 4 (Long-run cost). The equilibrium level to which a technology is developed in a competitive market increases with spillovers only up to a point and is the lowest for high spillovers. While an increase 


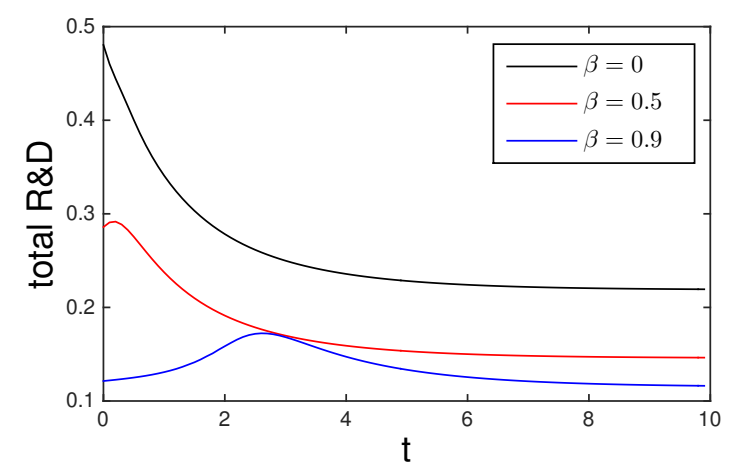

(a)

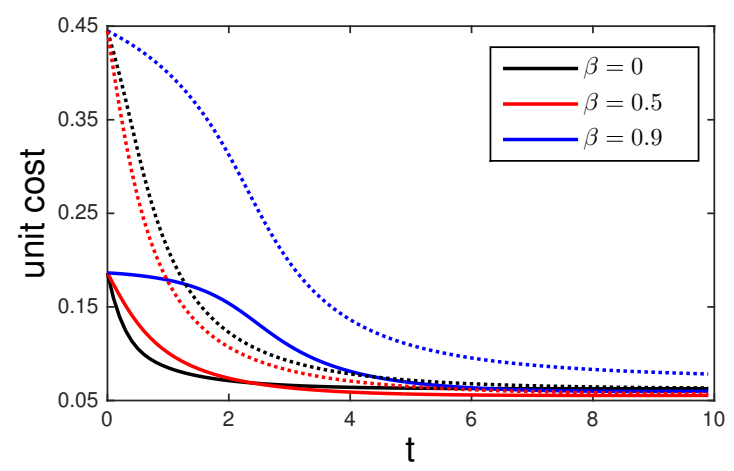

(c)

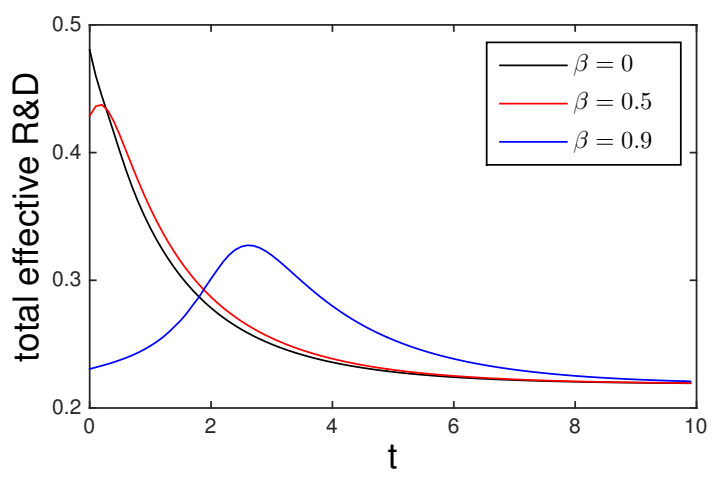

(b)

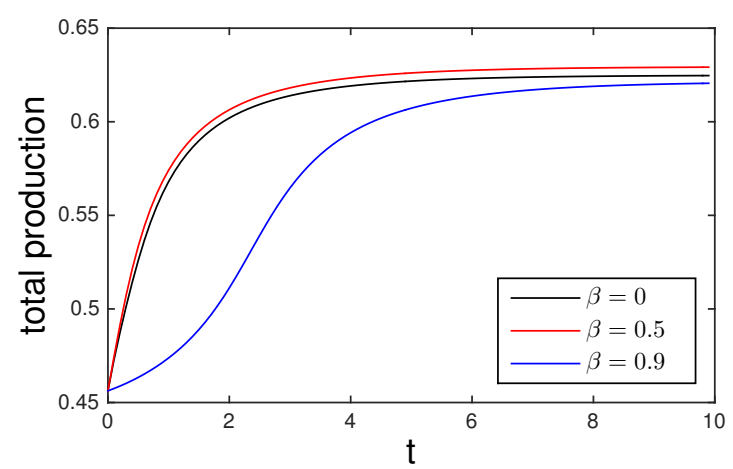

(d)

Figure 10: Time paths based on the drift vector field for varying levels of spillovers. In plot (c), the solid curves correspond to the leader and the dotted ones to the laggard. Initial state: $\left(c_{1}, c_{2}\right)=(0.19,0.44)$, parameters: $(\phi, \rho, \varepsilon)=(8,1,0.125)$.

in spillovers may improve the likelihood of a competitive market, it may at the same time reduce the level to which a technology is developed.

To get some insight into the effects of spillovers on firms at different stages of technological development, in Figure 10 we plot time paths for R\&D, production costs, and quantity produced. These are based on the drift vector fields presented earlier. As such, they neglect random shocks, but still provide us with some idea about how firms steer their R\&D and costs over time. We select an asymmetric initial position, $\left(c_{1}, c_{2}\right)=(0.19,0.44)$, which lies in Domain 1 (duopoly) for all the three different levels of spillovers considered.

Figure 10a shows total R\&D efforts by two competing firms over time for different levels of spillovers. We see a typical effect of increasing spillovers - the total industry R\&D efforts decrease as firms increasingly free-ride on each other. However, Figure 10b shows that due to larger complementarities between R\&D efforts at higher spillovers, the effective efforts of firm $i$ and firm $j,(1+\beta)\left(k_{i}+k_{j}\right)$, can be larger at higher spillovers despite the firms' lower de facto $\mathrm{R} \& \mathrm{D}$ efforts, $k_{i}+k_{j}$. For parameters in Figure $10 \mathrm{~b}$, this is indeed the case when spillovers increase from $\beta=0$ to $\beta=0.5$. While for $\beta=0.5$, the industry R\&D efforts are relatively lower at all times, the effective industry R\&D efforts are larger for most of the time. This explains why in the latter case, the unit costs converge to a lower long-run level than in the case with $\beta=0$. We see that among the three levels of spillovers, the industry R\&D efforts are comparably the lowest for $\beta=0.9$. At the beginning, the leader in the latter case invests very little as he free-rides on the efforts of the laggard. These smaller investments are not offset by higher spillovers, 
such that the effective efforts are much lower than in the other two cases. This changes over time as the leader himself starts to invest more when the laggard gradually reduces his efforts over time. However, as Figure 10c shows, lower effective investments at the beginning very much slow down the speed at which unit costs decrease. In the case of $\beta=0.9$, the unit costs of both the leader (solid line) and the laggard (dotted line) decrease much slower than in the other two cases. Moreover, the gap between the laggard and the leader also closes more slowly. These slower and lower reductions of costs as a consequence of smaller investments are the reason that the total quantity offered in the market is for $\beta=0.9$ at all times the lowest among the cases considered (see Figure 10d). The largest total quantity is offered for $\beta=0.5$, whereas the total quantity for $\beta=0$ is close to that for $\beta=0.5$, but slightly lower.

Calculations show that in the above example total profits increase with spillovers. This is the effect of higher complementarities in $R \& D$ outputs that allow for significant savings on $R \& D$ costs. However, consumers are not necessarily any better for it. As our comparisons indicate, there exists a threshold level of spillovers after which further increases in spillovers do not benefit consumers. At high spillovers, the free riding effect induces firms to invest less and the consequent lower production efficiency, to the detriment of consumers, also induces them to produce less. Of course, relative investment at different spillovers depends on parameters. Thus, in later Section 5, we calculate surpluses for a wide range of different parameter configurations to draw robust conclusions about the effect of spillovers on welfare.

\subsection{Equilibrium Dynamics II: Strained Market}

When we keep reducing the profit potential of a technology $(\phi)$ for a given discount rate $(\rho)$, the unstable steady state $S_{3}$ eventually enters the positive production region of state space (see Figure 11). This implies that technologies with an initial unit cost above the choke price are not developed further, while those with unit costs that allow immediate production are developed only if these unit costs are sufficiently low, such that they do not require "too much" R\&D effort to develop and maintain. In general, lowering $\phi$ for a given $\rho$ moves steady states $S_{3}$ and $S_{4}$ closer together. These has three consequences. First, it makes it less likely that some initial technology will be developed. Second, it reduces the level to which any technology will be developed (the long-run equilibrium production costs are higher). Last, it reduces the region of state space for which there is a eventually duopoly in the product market (the region between the $W_{1}^{S}$ and $W_{2}^{S}$ separatrices, i.e., Domain 1). When demand decreases or R\&D costs rise (recall that these define $\phi$ in Lemma 1), a much smaller lead is needed to induce the laggard to give up. Figure 11b indicates this for the case of $\beta=0.5$. Observe that neither firm develops further a technology which would require investments prior to production - the basin of attraction of $S_{4}$ is compressed and fully contained within the production area delimited by the $E_{1}$ and $E_{2}$ curves (compare with Figure 3).

Domain 1 is the smallest at low spillovers, where it can for sufficiently small $\phi$ even become compressed into a line. This happens for parameters in Figure 11a, where for low spillovers there are only two steady states - a nodal source $S_{3}$ and a saddle-point steady state $S_{1}^{*}$. The two saddles ( $S_{1}$ and $S_{2}$ ) and the nodal sink $\left(S_{4}\right)$ have colluded and formed a new steady state $S_{1}^{*}$. This new saddle has two manifolds - the unstable manifold $W_{1}^{U}$ and the stable manifold $W_{1}^{S}$ which lies on the diagonal of the state space. The implication of this is that the region of duopoly is compressed into a line segment which originates in $S_{3}$, passes through $S_{1}^{*}$ and approaches zero. Only for symmetric positions on this line, both firms keep producing and steer their unit costs towards the long-run equilibrium level of $S_{1}^{*} \cdot{ }^{25}$ However, any initial asymmetry induces the less efficient firm to gradually exit the market. At low spillovers, a small lead is enough to induce the laggard to give up. Observe how the diagonal acts like a repeller - on

\footnotetext{
${ }^{25}$ If an initial position happens to lie on the diagonal above $S_{1}^{*}$, firms find it optimal to decrease their efficiency towards a higher long-run level of unit costs that is less costly to maintain.
} 


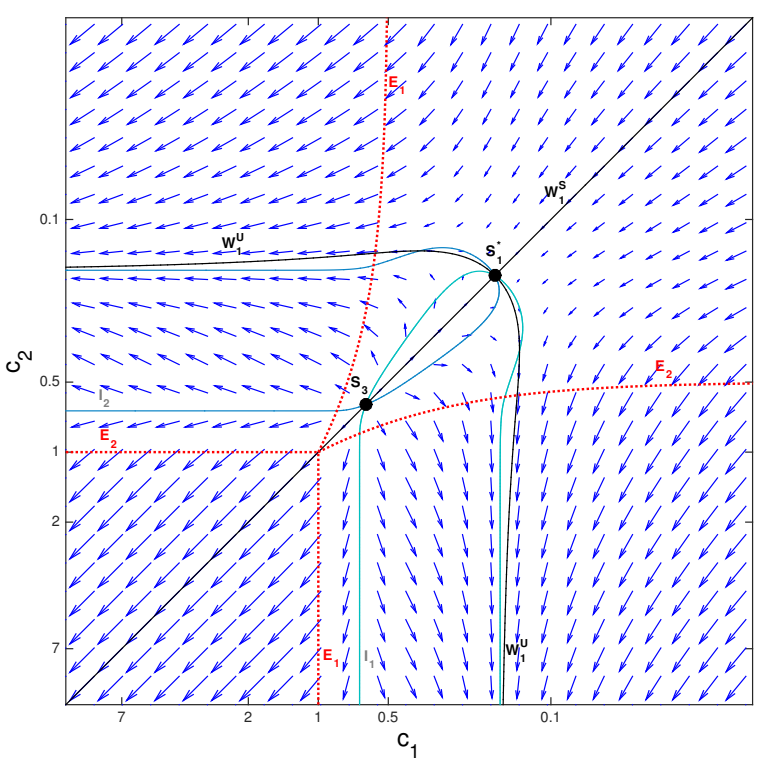

(a) $\beta=0.1$

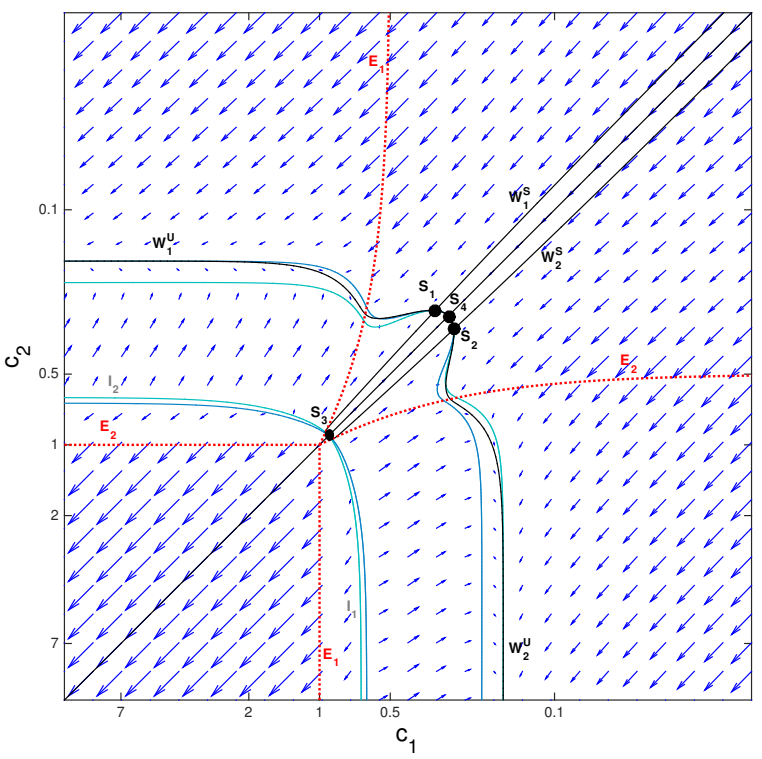

(c) $\beta=0.9$

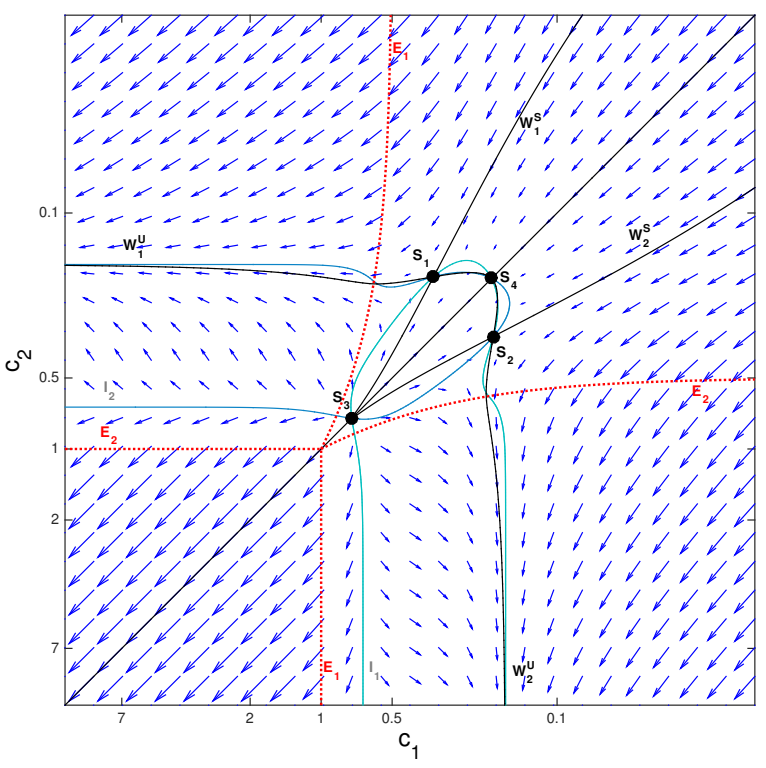

(b) $\beta=0.5$

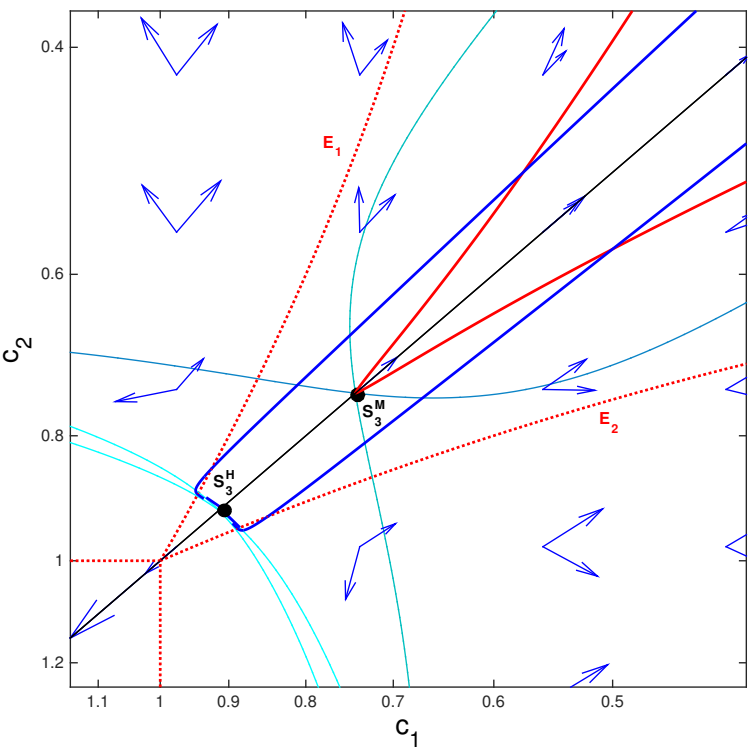

(d) $\beta=0.5$ vs $\beta=0.9$

Figure 11: Drift vector field for small (a), medium (b), and high (c) spillovers, $(\phi, \rho, \varepsilon)=(5,1,0.125)$.

each side of it, the motion is away from it. Clearly, with random shocks to costs, any symmetry in costs is only temporary, such that for most of the time one firm diverges out of the market.

It is noteworthy that for less favorable market and R\&D conditions, a complete asymmetry can emerge for low spillovers - initial asymmetries always lead to asymmetric outcomes (a firm with a cost advantage becomes a monopolist, whereas the other firm exits the market). This is different from the Promising Technology dynamics in the previous section, where Domain 1 was always a proper region, such that it was possible for firms to overcome (sufficiently small) asymmetries even at low spillovers (at least at lower unit costs away from the spike).

As spillovers increase, for a given value of other parameters, $S_{1}^{*}$ transforms into two saddles $\left(S_{1}\right.$ and $S_{2}$ ) and a nodal sink $\left(S_{4}\right)$. The region of duopoly becomes a proper region, as is the case for $\beta=0.5$ in 
Figure 11b. As spillovers grow, the two saddles $\left(S_{1}\right.$ and $\left.S_{2}\right)$ move aside and the region of duopoly grows larger. After some critical point of spillovers, however, the saddles start approaching each other and so the region of duopoly starts to contract. This effect is visible in Figure 11 when we compare the drift vector fields for $\beta=0.1, \beta=0.5$, and $\beta=0.9$.

Figure $11 \mathrm{~d}$, which shows a zoomed comparison between $\beta=0.5$ and $\beta=0.9$, indicates that this contraction of the duopoly region at higher spillovers is again not universal (recall Figure 9 ) as the region of duopoly for $\beta=0.9$ remains wider at larger levels of unit costs. All in all, our conclusion is similar as before - after a certain level, higher spillovers start to reduce the duopoly region as smaller investments of the unmotivated leader makes it harder for the laggard to catch up.

Result 5 (Strained market). When profit potential of a technology is low, low spillovers are most conducive to market monopolization. At low spillovers, even a slight cost advantage at any stage of development may suffice for a leader to squeeze a laggard out of the market. A sufficiently high level of spillovers may be needed to make a competitive market a possibility. There exists, however, a critical level of spillovers beyond which further increases in spillovers start favouring a monopolistic outcome.

\subsection{Equilibrium Dynamics III: Obsolete Technology}

Reducing $\phi$ for a given $\rho$, we eventually arrive at the situation in which demand is so low and/or the R\&D process so costly that both firms find it optimal to (eventually) leave the market. The drift vector field in Figure 12 shows that for all initial positions, unit costs of both firms diverge towards infinity. Firms might still invests in $R \& D$ at some smaller rate to retard the technical decay, but eventually both the R\&D and production will terminate and the firms will exit the market. ${ }^{26}$

In Figure 12a, $\rho=1$. In this case, the exit from the market is relatively fast, such that the difference between spillovers is very small. The difference is more pronounced at lower values of $\rho$, where firms in general have higher incentives to stay longer in the product market. Figure 12b compares the drift vector field at low spillovers (black arrows) to the one at high spillovers (blue arrows) when the discount rate is comparably low $(\rho=0.1)$. Observe that blue arrows are usually shorter (high spillovers slow down increases in price and delay the exit of firms) and with a slope closer to 1 (by keeping markets more symmetric, high spillovers favor competition in the product market). ${ }^{27}$ Both features are potentially of benefit to consumers.

Result 6 (Obsolete technology). When a technology is destined to leave the market, high spillovers delay the decay of a technology and thereby the exit of firms. This effect is stronger, the lower the discount rate.

\section{Welfare Effects of Spillovers}

To analyze the welfare effects of spillovers, we use the expected net present value of consumer surplus, producer surplus, and total surplus. To obtain the expected NPV of consumer surplus, we calculate the present values of the standard Marshallian consumer surplus for 1,000 stochastic paths of unit costs, all

\footnotetext{
${ }^{26}$ Utterback (1994) presents many examples of producers who prolonged the lifetime of their inferior technology through continuous innovations after the arrival of a superior technology. For instance, steam-powered saws were first used in the natural ice harvesting industry after the arrival of machine-made ice. Producers of gas lamps introduced many product innovations after Edison's invention of an electric bulb, including the Welsbach mantle that made them five times more efficient. Likewise, at the time when Kodak was introducing roll film cameras, several improvements in dry plate photography were developed (e.g., celluloid substitutes for glass, self-setting shutters, and small plate cameras).

${ }^{27}$ Arrows for $\beta=0.5$ (not shown) consistently appear between black and blue arrows throughout the relevant part of parameter space.
} 


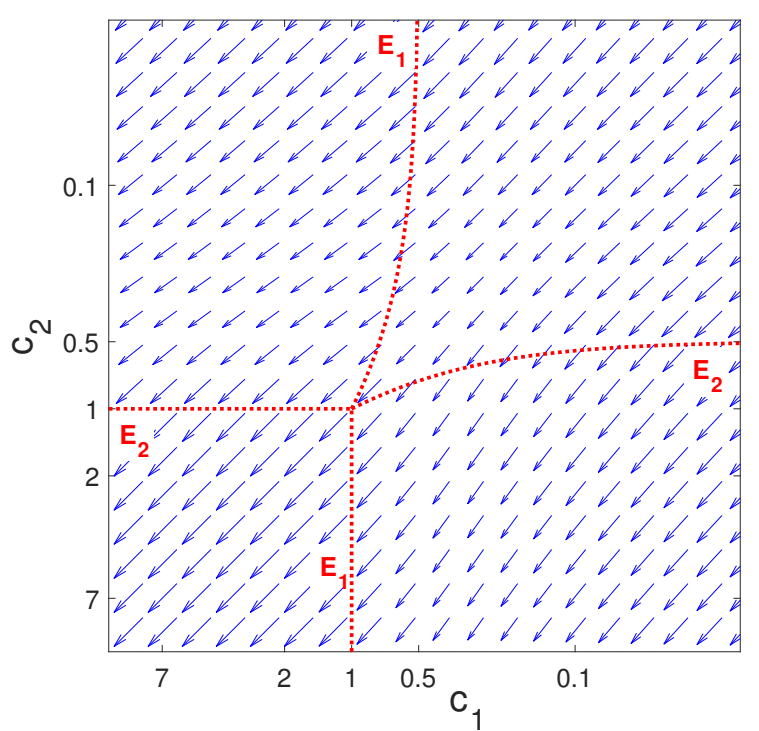

(a) $\beta=0.5$

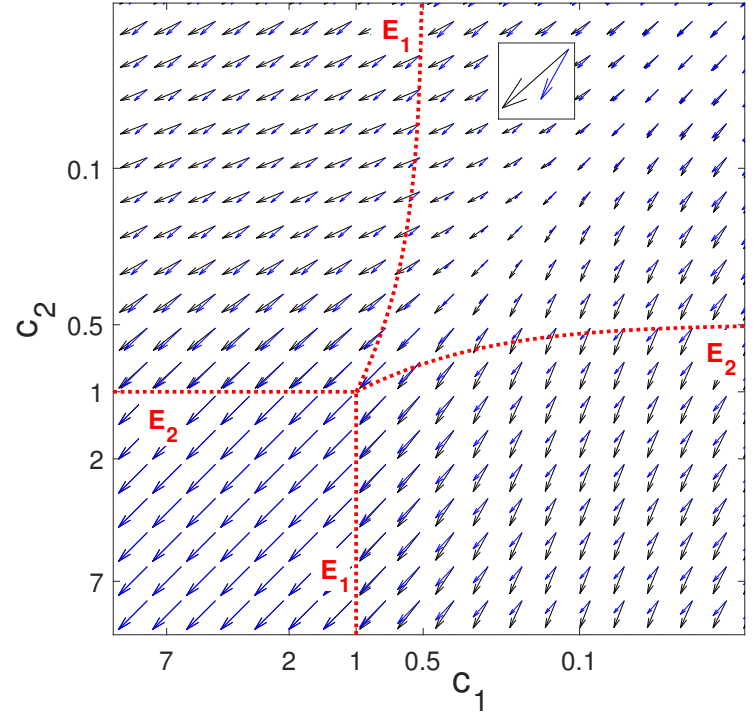

(b) $\beta=0.1$ (black) vs $\beta=0.9$ (blue)

Figure 12: Drift vector field when exiting the market is always optimal. In plot $(\mathrm{a}),(\phi, \rho, \varepsilon)=$ $(3,1,0.125)$. In plot $(b),(\phi, \rho, \varepsilon)=(2,0.1,0.125)$.

starting in the same initial point, and take their mean value. ${ }^{28}$ We calculate producer surplus as the sum of firms' expected profits. We obtain the latter by evaluating firms' value functions in the initial point $\left(c_{1}(0), c_{2}(0)\right)$. Finally, the total surplus is obtained as the sum of the first two surpluses.

We consider three different levels of spillovers: low $\left(\beta_{L}=0.1\right)$, medium $\left(\beta_{M}=0.5\right)$, and high $\left(\beta_{H}=0.9\right)$. We fix noise $\varepsilon$ at a low level (usually $\varepsilon=0.125$ ), ${ }^{29}$ such that there is some uncertainty in the evolution of production costs, but that most of it is still due to firms' $R \& D$. We then investigate a range of values for the profit potential, $\phi \in(0,15]$, and for the rescaled discount rate, $\rho \in[0.1,5]$. As $\rho=r / \delta$ (recall Lemma 1$)$, the last interval includes a wide range of possible values for the discount rate $(r)$ and technology depreciation rate $(\delta)$. For instance, with a $5 \%$ discount rate $(r=0.05)$, as low as $1 \%$ $(\delta=0.01)$ and as high as $50 \%(\delta=0.5)$ depreciation rates are included.

We report results for a subset of different parameter configurations considered in Table 1 and Table 2. The two tables differ only in the assumed initial unit costs of firms. In Table 1, we assume high initial unit costs, $\left(c_{1}(0), c_{2}(0)\right)=(1.35,1.35)$, such that $\mathrm{R} \& \mathrm{D}$ necessarily precedes production, while in Table 2 , we assume low initial unit costs, $\left(c_{1}(0), c_{2}(0)\right)=(0.37,0.37)$, such that production is immediate.

To assess the importance of initial asymmetries, we also simulate the model for different relative initial unit costs of firms. We are interested in how welfare measures relate to spillovers when we vary the initial difference in unit costs, $d=c_{1}-c_{2}$. For this, we pick up an initial point on the diagonal of the state space $(d=0)$ and vary the costs such that their sum remains unchanged (i.e., $c_{1}+c_{2}=$ const.). Two

\footnotetext{
${ }^{28}$ As the game is infinite, we need to truncate the time interval of simulations. We impose that a simulation stops once a surplus increment over a time step has fallen below $1 \times 10^{-6}$. If the boundary of the state space is reached within the simulated time interval, we impose that from the moment the boundary is reached, the instantaneous surplus remains constant.

${ }^{29}$ The case with low spillovers is the hardest one to integrate numerically. For small values of $\varepsilon$, the spike in the policy function can increase and sharpen dramatically, posing problems for the stability of the numerical scheme. To make consistent comparisons, we always use the same noise level for all spillover levels, given the value of other parameters. Due to the constraint at low spillovers, we report the results for $\varepsilon=0.125$, except for the following parameter configurations where we necessarily use a higher value: $(\phi, \rho)=(5,0.1)$ with $\varepsilon=0.250,(\phi, \rho)=(15,0.5)$ with $\varepsilon=0.233,(\phi, \rho)=(8,0.5)$ with $\varepsilon=0.129,(\phi, \rho)=(15,1)$ with $\varepsilon=0.196$. We address the robustness of results to different values of $\varepsilon$ at the end of the section.
} 
such "iso-sum" loci (ISL) are shown in Figure 9. ${ }^{30} I S L_{1}$ intersects the diagonal at $c_{1}=c_{2}=1.35>1$, whereas $I S L_{2}$, which corresponds to a lower sum of unit costs, intersects it at $c_{1}=c_{2}=0.37<1$. For every initial point on the loci, we then calculate the NPV of surpluses in the usual way. ${ }^{31}$ Figure 13 shows results for two different parameter configurations. Note that the point $d=0$ in the first two rows in Figure 13 corresponds to Table 1, while the one in the last two rows corresponds to Table 2.

When it comes to producer surplus, the ranking of spillovers is unambiguous, at least for symmetric initial states. Both in Table 1 and in Table 2, producer surplus increases with spillovers for all parameter configurations. This is due to a large cost-saving effect that high complementarities in R\&D bring about. For more asymmetric initial states, however, lower spillovers can be preferred (see the second row in Figure 13). There are two countervailing effects at work. At low spillovers, cost reductions are costlier for a firm as it has to mostly rely on its own R\&D to reduce production costs. This reduces the firm's expected profits. On the other hand, when spillovers are low, the leader is more likely to be able to keep its advantage and secure itself a monopoly position in the future. This effect increases expected profits. It is stronger, the lower the discount rate and so the higher the importance that is attached to future higher mark-ups in the product market relative to higher current R\&D costs induced by lower spillovers.

When it comes to consumer surplus, the story is more involved. Consider first the situation in which initial costs are high and symmetric (see Table 1). Recall that it is low spillovers for which we usually observe the highest $R \& D$ efforts by firms battling for market dominance (cf. the spike in Figure 6). This is of benefit to consumers as it is then when production costs fall fastest. On the other hand, low spillovers are associated with the highest probability of a monopolized market, which is not of benefit to consumers. The final result will therefore depend on which effect is stronger.

Consider the first three rows in Table 1 , for $\rho=0.1$. There, the profit potential is very high, such that the steady state $S_{3}$ and the spike at $\beta_{L}$ are considerably above the initial point. In these cases, the market is so profitable that it would be difficult for any firm to squeeze the other one out of it, given the initial cost. Consequently, the $\mathrm{R} \& \mathrm{D}$ efforts at $\beta_{L}$ are not much higher than at $\beta_{M}$ in the vicinity of $c_{0}$. For most of the time, $\beta_{M}$ is associated with higher total effective R\&D efforts and so greater production. Medium spillovers dominate. This changes when $\phi$ falls and the spike in the policy function at $\beta_{L}$ moves closer to the initial point. For $\phi=3, \beta_{L}$ is characterized by huge investments of firms, each trying to secure its position in the market, and leads to the highest consumer surplus. When profit potential is sufficiently small given the discount rate (e.g., $\phi=2$ for $\rho=0.1$ ), firms optimally abstain from developing the technology as future expected sales are not sufficient to cover for losses incurred during the initial periods when firms would invest but not produce yet. In this case, expected consumer surplus is zero as there is no production. For other values of $\rho$, the story is similar. For instance, when $(\phi, \rho)=(8,1)$, low spillovers are characterized by high R\&D efforts, but which are nevertheless not high enough to offset an induced higher probability of monopoly. Hence, medium spillovers perform best (see also the first row in Figure 13). This changes as $\phi$ grows larger (or $\rho$ lower) and resulting larger predatory investments at low spillovers lead to considerably faster cost reductions.

In sum, when it comes to consumer surplus, the choice is usually between low and medium spillovers. However, as the second row in Figure 13 shows, low spillovers become ever less desirable for consumers

\footnotetext{
${ }^{30}$ The figure shows only the part of loci above the diagonal. As loci have a diagonal line of symmetry, the second part is computationally redundant.

${ }^{31}$ It is a standard feature of Cournot equilibrium that as long as both firms produce, industry output, price, and consumer surplus remain unchanged for exogenous changes in marginal costs that preserve their sum (see Salant and Shaffer, 1999). However, in our model, changes in initial unit costs (which equal marginal costs) induce strategic responses of firms, such that all these variables do change in course of time.
} 
Table 1: Net Present Value of Consumer Surplus, Producer Surplus, and Total Surplus for High Initial Value of Unit Cost

\begin{tabular}{|c|c|c|c|c|c|c|c|c|c|c|c|c|}
\hline \multicolumn{13}{|c|}{ Consumer Surplus } \\
\hline \multirow[b]{2}{*}{$\phi$} & \multicolumn{3}{|c|}{$\rho=0.1$} & \multicolumn{3}{|c|}{$\rho=0.5$} & \multicolumn{3}{|c|}{$\rho=1$} & \multicolumn{3}{|c|}{$\rho=2$} \\
\hline & $\beta_{L}$ & $\beta_{M}$ & $\beta_{H}$ & $\beta_{L}$ & $\beta_{M}$ & $\beta_{H}$ & $\beta_{L}$ & $\beta_{M}$ & $\beta_{H}$ & $\beta_{L}$ & $\beta_{M}$ & $\beta_{H}$ \\
\hline 15 & 0.684 & 0.937 & 0.686 & 0.277 & 0.315 & 0.275 & 0.141 & 0.141 & 0.129 & 0.070 & 0.038 & 0.034 \\
\hline 8 & 1.016 & 1.470 & 1.007 & 0.289 & 0.276 & 0.241 & 0.054 & 0.060 & 0.054 & 0.000 & 0.000 & 0.001 \\
\hline 5 & 1.025 & 1.381 & 0.961 & 0.062 & 0.107 & 0.113 & 0.000 & 0.001 & 0.000 & 0.000 & 0.000 & 0.000 \\
\hline 3 & 1.705 & 1.254 & 1.020 & 0.000 & 0.000 & 0.000 & 0.000 & 0.000 & 0.000 & 0.000 & 0.000 & 0.000 \\
\hline 2 & 0.000 & 0.000 & 0.000 & 0.000 & 0.000 & 0.000 & 0.000 & 0.000 & 0.000 & 0.000 & 0.000 & 0.000 \\
\hline
\end{tabular}

Producer Surplus

\begin{tabular}{|c|c|c|c|c|c|c|c|c|c|c|c|c|}
\hline \multirow[b]{2}{*}{$\phi$} & \multicolumn{3}{|c|}{$\rho=0.1$} & \multicolumn{3}{|c|}{$\rho=0.5$} & \multicolumn{3}{|c|}{$\rho=1$} & \multicolumn{3}{|c|}{$\rho=2$} \\
\hline & $\beta_{L}$ & $\beta_{M}$ & $\beta_{H}$ & $\beta_{L}$ & $\beta_{M}$ & $\beta_{H}$ & $\beta_{L}$ & $\beta_{M}$ & $\beta_{H}$ & $\beta_{L}$ & $\beta_{M}$ & $\beta_{H}$ \\
\hline 15 & 1.826 & 1.930 & 1.955 & 0.223 & 0.282 & 0.298 & 0.054 & 0.094 & 0.106 & 0.006 & 0.019 & 0.025 \\
\hline 8 & 1.392 & 1.644 & 1.694 & 0.055 & 0.154 & 0.185 & 0.016 & 0.027 & 0.039 & 0.000 & 0.000 & 0.000 \\
\hline 5 & 0.813 & 1.229 & 1.304 & 0.014 & 0.034 & 0.068 & 0.000 & 0.000 & 0.000 & 0.000 & 0.000 & 0.000 \\
\hline 3 & 0.144 & 0.362 & 0.695 & 0.000 & 0.000 & 0.000 & 0.000 & 0.000 & 0.000 & 0.000 & 0.000 & 0.000 \\
\hline 2 & 0.000 & 0.000 & 0.000 & 0.000 & 0.000 & 0.000 & 0.000 & 0.000 & 0.000 & 0.000 & 0.000 & 0.000 \\
\hline
\end{tabular}

Total Surplus

\begin{tabular}{|c|c|c|c|c|c|c|c|c|c|c|c|c|}
\hline \multirow[b]{2}{*}{$\phi$} & \multicolumn{3}{|c|}{$\rho=0.1$} & \multicolumn{3}{|c|}{$\rho=0.5$} & \multicolumn{3}{|c|}{$\rho=1$} & \multicolumn{3}{|c|}{$\rho=2$} \\
\hline & $\beta_{L}$ & $\beta_{M}$ & $\beta_{H}$ & $\beta_{L}$ & $\beta_{M}$ & $\beta_{H}$ & $\beta_{L}$ & $\beta_{M}$ & $\beta_{H}$ & $\beta_{L}$ & $\beta_{M}$ & $\beta_{H}$ \\
\hline 15 & 2.509 & 2.867 & 2.641 & 0.500 & 0.597 & 0.573 & 0.195 & 0.235 & 0.236 & 0.076 & 0.057 & 0.058 \\
\hline 8 & 2.408 & 3.114 & 2.701 & 0.344 & 0.430 & 0.425 & 0.069 & 0.087 & 0.093 & 0.001 & 0.001 & 0.001 \\
\hline 5 & 1.838 & 2.609 & 2.265 & 0.076 & 0.141 & 0.181 & 0.000 & 0.001 & 0.001 & 0.000 & 0.000 & 0.000 \\
\hline 3 & 1.849 & 1.616 & 1.715 & 0.000 & 0.000 & 0.000 & 0.000 & 0.000 & 0.000 & 0.000 & 0.000 & 0.000 \\
\hline 2 & 0.000 & 0.001 & 0.000 & 0.000 & 0.000 & 0.000 & 0.000 & 0.000 & 0.000 & 0.000 & 0.000 & 0.000 \\
\hline
\end{tabular}

Spillovers: $\beta_{L}=0.1, \beta_{M}=0.5, \beta_{H}=0.9$; initial cost: $\left(c_{1}, c_{2}\right)=(1.35,1.35)$.

when firms become more asymmetric. ${ }^{32,33}$ The reason is that low spillovers make it harder for the laggard to catch up with the leader, which is through softer competition in the product market detrimental to consumers. For more asymmetric initial costs, medium spillovers are generally preferred. However rare, there exist specific parameter configurations for which high spillovers bring about the largest consumer surplus. This happens when high spillovers are needed for firms to develop the technology at all (as in Figure 9), or when a much lower probability of monopoly at high spillovers offsets concomitant comparably lower R\&D efforts due to the free-riding effect (e.g., $(\phi, \rho)=(5,0.5)$ in Table 1).

When initial costs are low (see Table 2 ) and firms find it optimal to stay in the market ( $\phi$ is sufficiently high for a given $\rho$ ), medium spillovers generally bring about the highest consumer surplus. With lower unit costs and production already in place, preemptive and predatory stimuli for firms at low spillovers have dissipated, such that medium spillovers are usually associated with the highest effective R\&D efforts, lowest costs, and thereby largest production. High spillovers most often perform worst. When initial production costs already permit profitable production, the free-riding effects of high spillovers are even

\footnotetext{
${ }^{32}$ Surplus usually starts increasing beyond a certain point of asymmetry as there what we primarily do is reduce the initial production cost of a (future) monopolist.

${ }^{33}$ At the end of the section, we argue that higher uncertainty $(\varepsilon)$ also makes low spillovers comparably less desirable. The reason is that by increasing $\varepsilon$, the policy function becomes smoother and the spike at low spillovers gradually disappears.
} 
Table 2: Net Present Value of Consumer Surplus, Producer Surplus, and Total Surplus for Low Initial Value of Unit Cost

\begin{tabular}{|c|c|c|c|c|c|c|c|c|c|c|c|c|}
\hline \multicolumn{13}{|c|}{ Consumer Surplus } \\
\hline \multirow[b]{2}{*}{$\phi$} & \multicolumn{3}{|c|}{$\rho=0.1$} & \multicolumn{3}{|c|}{$\rho=0.5$} & \multicolumn{3}{|c|}{$\rho=1$} & \multicolumn{3}{|c|}{$\rho=2$} \\
\hline & $\beta_{L}$ & $\beta_{M}$ & $\beta_{H}$ & $\beta_{L}$ & $\beta_{M}$ & $\beta_{H}$ & $\beta_{L}$ & $\beta_{M}$ & $\beta_{H}$ & $\beta_{L}$ & $\beta_{M}$ & $\beta_{H}$ \\
\hline 15 & 0.690 & 0.964 & 0.668 & 0.306 & 0.346 & 0.308 & 0.174 & 0.178 & 0.171 & 0.075 & 0.076 & 0.073 \\
\hline 8 & 1.040 & 1.507 & 1.046 & 0.347 & 0.351 & 0.333 & 0.144 & 0.145 & 0.130 & 0.053 & 0.053 & 0.049 \\
\hline 5 & 1.082 & 1.465 & 1.057 & 0.277 & 0.277 & 0.228 & 0.092 & 0.093 & 0.088 & 0.037 & 0.037 & 0.036 \\
\hline 3 & 1.523 & 1.653 & 1.243 & 0.091 & 0.097 & 0.110 & 0.049 & 0.049 & 0.050 & 0.030 & 0.030 & 0.029 \\
\hline 2 & 0.110 & 0.115 & 0.132 & 0.056 & 0.058 & 0.058 & 0.041 & 0.042 & 0.042 & 0.028 & 0.028 & 0.027 \\
\hline
\end{tabular}

Producer Surplus

\begin{tabular}{|c|c|c|c|c|c|c|c|c|c|c|c|c|}
\hline \multirow[b]{2}{*}{$\phi$} & \multicolumn{3}{|c|}{$\rho=0.1$} & \multicolumn{3}{|c|}{$\rho=0.5$} & \multicolumn{3}{|c|}{$\rho=1$} & \multicolumn{3}{|c|}{$\rho=2$} \\
\hline & $\beta_{L}$ & $\beta_{M}$ & $\beta_{H}$ & $\beta_{L}$ & $\beta_{M}$ & $\beta_{H}$ & $\beta_{L}$ & $\beta_{M}$ & $\beta_{H}$ & $\beta_{L}$ & $\beta_{M}$ & $\beta_{H}$ \\
\hline 15 & 1.936 & 2.006 & 2.019 & 0.313 & 0.346 & 0.355 & 0.127 & 0.150 & 0.156 & 0.049 & 0.061 & 0.065 \\
\hline 8 & 1.611 & 1.792 & 1.820 & 0.201 & 0.268 & 0.285 & 0.075 & 0.107 & 0.118 & 0.038 & 0.046 & 0.049 \\
\hline 5 & 1.143 & 1.453 & 1.494 & 0.113 & 0.188 & 0.221 & 0.065 & 0.081 & 0.089 & 0.035 & 0.038 & 0.040 \\
\hline 3 & 0.412 & 0.713 & 1.081 & 0.079 & 0.092 & 0.107 & 0.053 & 0.056 & 0.058 & 0.033 & 0.034 & 0.035 \\
\hline 2 & 0.097 & 0.105 & 0.124 & 0.067 & 0.070 & 0.072 & 0.050 & 0.051 & 0.052 & 0.033 & 0.033 & 0.033 \\
\hline
\end{tabular}

Total Surplus

\begin{tabular}{|c|c|c|c|c|c|c|c|c|c|c|c|c|}
\hline \multirow[b]{2}{*}{$\phi$} & \multicolumn{3}{|c|}{$\rho=0.1$} & \multicolumn{3}{|c|}{$\rho=0.5$} & \multicolumn{3}{|c|}{$\rho=1$} & \multicolumn{3}{|c|}{$\rho=2$} \\
\hline & $\beta_{L}$ & $\beta_{M}$ & $\beta_{H}$ & $\beta_{L}$ & $\beta_{M}$ & $\beta_{H}$ & $\beta_{L}$ & $\beta_{M}$ & $\beta_{H}$ & $\beta_{L}$ & $\beta_{M}$ & $\beta_{H}$ \\
\hline 15 & 2.626 & 2.970 & 2.687 & 0.619 & 0.692 & 0.662 & 0.301 & 0.327 & 0.327 & 0.124 & 0.137 & 0.138 \\
\hline 8 & 2.651 & 3.299 & 2.866 & 0.549 & 0.619 & 0.618 & 0.220 & 0.252 & 0.248 & 0.091 & 0.099 & 0.098 \\
\hline 5 & 2.225 & 2.919 & 2.551 & 0.390 & 0.465 & 0.449 & 0.157 & 0.175 & 0.177 & 0.072 & 0.075 & 0.076 \\
\hline 3 & 1.935 & 2.366 & 2.323 & 0.171 & 0.189 & 0.216 & 0.102 & 0.105 & 0.108 & 0.063 & 0.064 & 0.064 \\
\hline 2 & 0.206 & 0.221 & 0.256 & 0.123 & 0.128 & 0.130 & 0.091 & 0.093 & 0.094 & 0.060 & 0.061 & 0.061 \\
\hline
\end{tabular}

Spillovers: $\beta_{L}=0.1, \beta_{M}=0.5, \beta_{H}=0.9$; initial cost: $\left(c_{1}, c_{2}\right)=(0.37,0.37)$.

worse than at high initial costs, as then once high $R \& D$ efforts of firms in anticipation of entering the product market have subsided. Hence, high spillovers are usually associated with the lowest R\&D efforts and the highest long-run equilibrium unit costs. As they also have the narrowest duopoly region at low costs (cf. Figure 9), asymmetries do not work in their favor anymore (see the last two rows in Figure 13). A situation in which we often find high spillovers perform best is when firms find it optimal to leave the market due to low profitability that makes it too expensive for them to maintain the technology in the face of its continuous depreciation (e.g., $(\phi, \rho)=(2,0.1)$ in Table 2). In such cases, high spillovers lead to slower reductions in production as they keep unit costs more symmetric (as in Figure 12b). Though, usually in such cases, the difference between spillovers is comparably low.

Consider now total surplus. When initial costs are high, Figure 13 indicates that low spillovers are least desirable from a social standpoint. In the second row, high consumer surplus, induced by large investments of initially more or less symmetric firms, is more than offset by comparably lower profits at low spillovers. However, this result very much depends on particular parameter values that make consumer surplus small relative to producer surplus. As Table 1 shows, there are parameter configurations for which low spillovers perform best (e.g., $(\phi, \rho)=(3,0.1)$ or $(\phi, \rho)=(15,2))$. Since firms that are not too different from each other always prefer high spillovers, we can conclude that for low spillovers to be 
$I S L_{1},(\phi, \rho)=(8,1)$
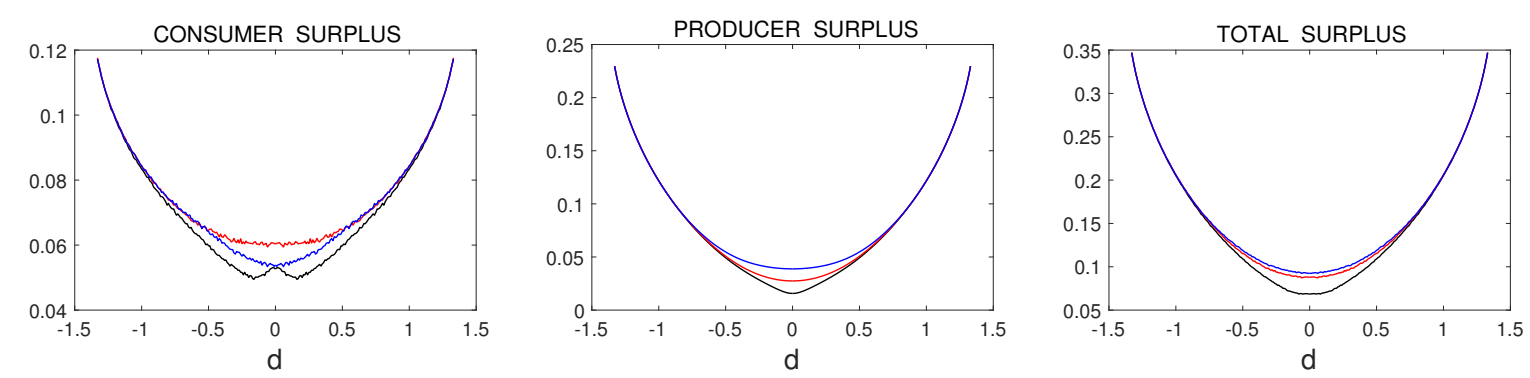

$I S L_{1},(\phi, \rho)=(8,0.5)$
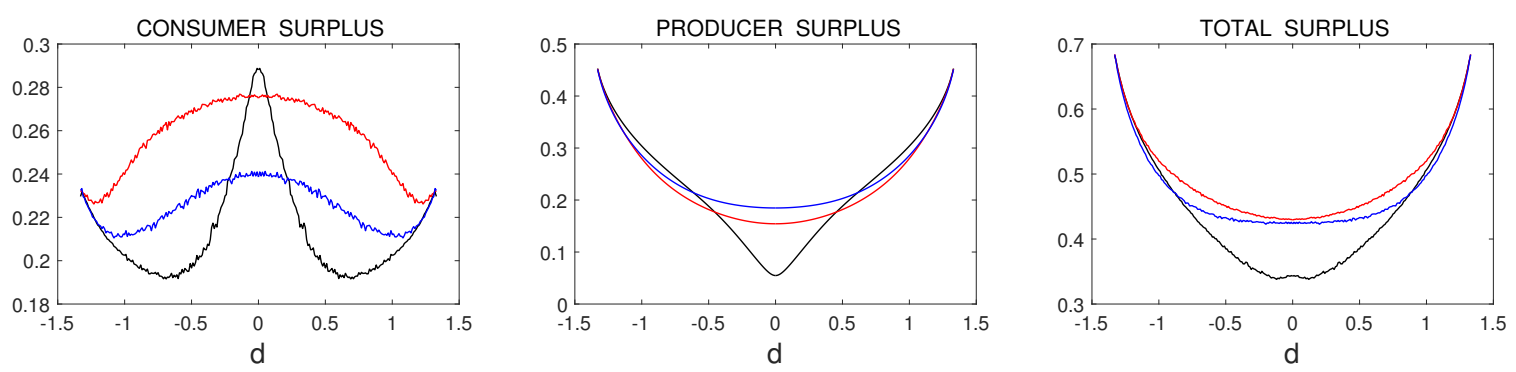

$$
I S L_{2},(\phi, \rho)=(8,1)
$$
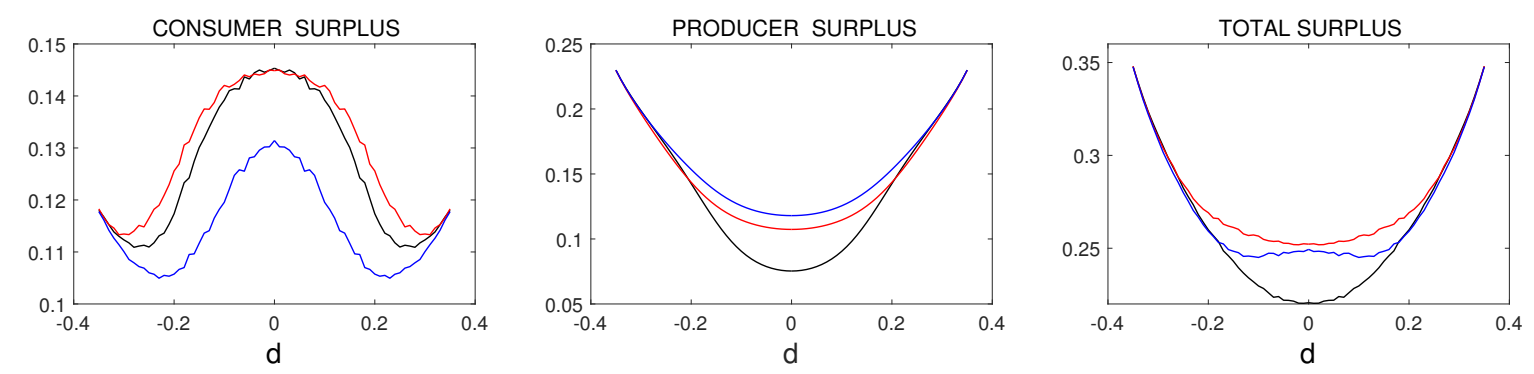

$$
I S L_{2},(\phi, \rho)=(8,0.5)
$$
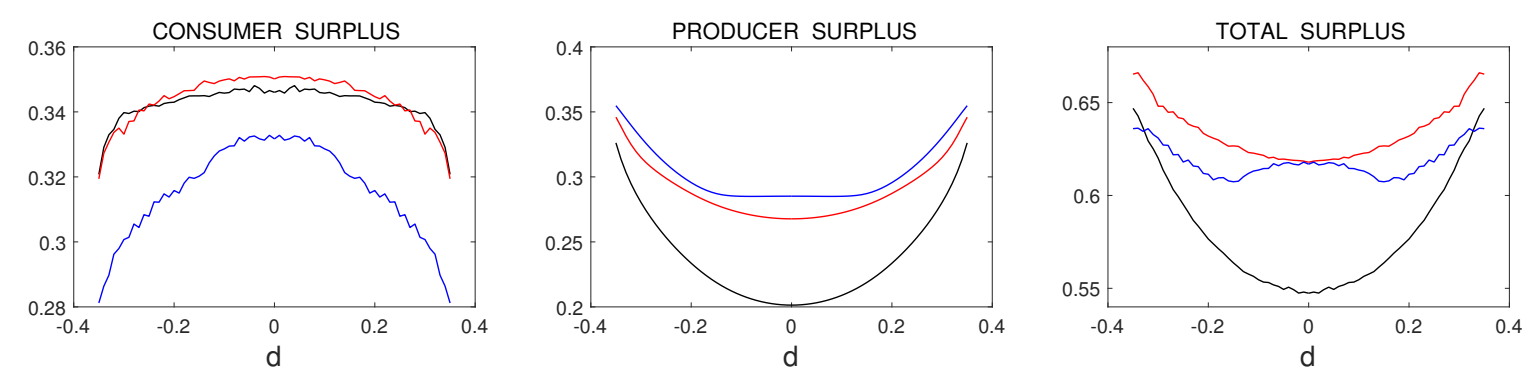

Figure 13: Net present value of consumer surplus, producer surplus, and total surplus for different levels of initial asymmetry; $\beta=0.1$ (black), $\beta=0.5$ (red), and $\beta=0.9$ (blue). 
socially preferred, a necessary, but not sufficient, condition is that they are preferred by consumers. We already know that with lower initial costs, low and high spillovers become less desirable for consumers. It is then of no surprise that for low initial costs, medium spillovers most frequently lead to the highest total surplus (see Table 2). This is true as long as $\phi$ is not so low that firms find it optimal to exit the market, in which case high spillovers usually lead to the largest total surplus.

A notable observation when comparing surpluses for high and low initial costs is that with lower initial costs, low spillovers become progressively less desirable. In Table 2, they lead to the lowest total surplus for all parameter values.

We see that consumers and firms usually have conflicting interests. While consumers usually prefer low or medium spillovers, firms prefer high spillovers. We therefore expect to find them on opposite sides of a policy debate.

Result 7 (Welfare). Consumers prefer a rapid fall in unit costs, which can be brought about by low spillovers between nearly symmetric firms leading to increased competition, or by medium spillovers between more asymmetric firms. High spillovers have a tendency to slow down the rate of decrease of unit costs. In contrast to this, firms usually prefer high spillovers, as these decrease their $R \& D$ costs.

When technology is relatively developed and associated production costs comparably low, low spillovers are socially the least preferred option, as they lead with high probability to monopolies. When technology is old and firms are leaving the market, high spillovers are usually the most preferred option. But in many situations, the result is non-monotonic, and an intermediate level of spillovers is socially preferred.

Our conclusions in this section are robust to different levels of uncertainty in $R \& D$, as long as the value of $\varepsilon$ remains low. As explained in the previous section, as $\varepsilon$ grows large, the policy function becomes ever smoother. In particular, the spike in the policy function at low spillovers gradually disappears, and with it any benefits accruing to consumers from associated rapid reductions in production costs. Therefore, for high $\varepsilon$, low spillovers are (eventually) dominated by medium spillovers with regard to consumer surplus, producer surplus, and total surplus. However, they are not necessarily dominated by high spillovers which still come with significant free-riding effects. ${ }^{34}$

\section{Summary and concluding remarks}

In this paper, we study feedback Nash equilibria of a dynamic game in which firms enhance their production efficiency through R\&D endeavors. Firms' product market participation constraints are explicitly taken into account. As a result, $R \& D$ efforts and production do not necessarily coexist at all times. In particular, $R \& D$ efforts can precede production, as it holds for the development phase of great many new technologies in practice. Consequently, we are for the first time able to study not only how spillovers affect investments in existing markets, but also how they affect the formation of markets and the evolution of their structure over time. We show that these effects can be of opposite sign - while an increase in spillovers may improve the likelihood of a technology being developed to the production stage and/or the future market being competitive, it may at the same time reduce the level to which a technology is developed in the long run.

\footnotetext{
${ }^{34}$ As an example, consider $(\phi, \rho)=(8,0.5)$ with varying levels of $\varepsilon$. According to Table 1 , when $\varepsilon$ is low $(\varepsilon=0.129)$, $\beta_{L}$ performs best in consumer surplus (due to rapid investment), but worst in producer surplus and total surplus. For $\varepsilon=0.5$, consumer surplus for $\beta_{L}, \beta_{M}$ and $\beta_{H}$ is $0.232,0.243$ and 0.206 , respectively. The corresponding producer surplus is 0.141 , 0.203 and 0.224 , while total surplus is $0.374,0.446$ and 0.429 . Higher uncertainty has removed the spike in the policy function and has thereby made low spillovers fall behind medium ones in consumer surplus, while in producer surplus and total surplus, the ranking is still unchanged, and remains so even for $\varepsilon=2$.
} 
We show that the pro-competitive effect of large spillovers, previously indicated in the literature, is not universally true. When initial production costs are relatively high, the likelihood of a competitive market increases with spillovers. This is not necessarily the case at later stages of technological development. When the leader progresses on the development ladder, his incentives to exert further R\&D efforts can be at high spillovers rather low as innovations can start to benefit the laggard relatively more than the leader himself. This obstructs the laggard's efforts to catch up with the leader through free-riding. Consequently, a smaller lag can induce the laggard to exit the market at higher spillovers. To the extent that we may associate weak patents with high spillovers, we can say that weak patents make R\&D results easier to copy, but if they also lead to less patents being taken out by innovators, thereby reducing the knowledge base available for imitation, they can well have a contra-competitive effect.

We find that in general duopoly arises in the product market only if initial asymmetries between the firms are not too large. If spillovers are low and initial unit costs relatively high, we find that a fierce $R \& D$ race starts, ending in duopoly only for almost completely symmetrical firms. There, a small cost advantage of one firm leads to a behavior that can be considered predatory: the leader exerts high R\&D efforts which are profitable in that they induce the laggard to give up. However, when firms start from a perfectly symmetric situation, their behavior resembles a preemption race: each firm invests a lot trying to win the race in which a small lead suffices for gaining a monopoly position, resulting in very fast technology development. The duopoly in the product market is characterized by the regression toward the mean phenomenon: asymmetries between the firms tend to vanish over time.

We find that larger random shocks to firms' production costs are increasing the likelihood that a technology will be developed further as firms are stimulated by the possibility of a favorable shock to their production costs in the future more than they are discouraged by the possibility of an unfavorable shock. They rather invest more today than be sorry later for giving up too early. Uncertainty also increases the likelihood that the product market will be competitive as the chance of a larger favorable shock in the future increases the perseverance of the laggard.

We show that the relation between spillovers, R\&D efforts, and surpluses depends both on the relative as well as the absolute level of competing technologies. Consumers usually prefer low or medium spillovers, while firms (as long as one does not have a significant advantage) prefer high spillovers. The total surplus pretty much depends on the relative size of consumer surplus and producer surplus at a given parameter configuration. However, when technology is relatively developed and associated production costs comparably low, low spillovers are usually socially the least preferred option. If anywhere, low spillovers can be socially desirable at high initial costs when they have a potential to induce firms to invest a lot in a race to secure their position in the future product market. This, for instance, differs from Acemoglu and Akcigit (2012) who propose a relatively stronger protection of intellectual property rights at later stages of development.

In the future we plan to extend the global framework to different forms of R\&D cooperatives and consider their desirability at different levels of spillovers. Several other interesting venues for future research open up as a consequence of our analysis. Our model is focused on a market for a single product and so does not consider a possibility that firms can simultaneously work on several products of different levels of substitution in the product market. In practice, for many high-tech firms a viable response to imitators seems to be introducing new versions of existing products. Moreover, the kind of technologies pursued can affect imitation capabilities and firms can intentionally make their research tracks more or less complementary to those of their competitors. This endogenous determination of $\mathrm{R} \& \mathrm{D}$ complementarities and absorption capacities is an interesting, though challenging, alternative to the current exogenous formulation of spillovers. 


\section{Appendices}

\section{A Proof of Lemma 1}

A scaled variable or parameter is distinguished by a tilde. Define the scaled variables by the following conversion equations: $q_{i}=A \tilde{q}_{i}, k_{i}=\frac{A}{\sqrt{b}} \tilde{k}_{i}, c_{i}=A \tilde{c}_{i}, \pi_{i}=A^{2} \tilde{\pi}_{i}, B_{i}(t)=\delta^{-1 / 2} \tilde{B}_{i}(\tilde{t})$ for $i=1,2$, as well as $t=\tilde{t} / \delta$. Here the $\tilde{B}_{i}$ for $i=1,2$ are independent standard Wiener processes. Introduce new parameters

$$
\phi=\frac{A}{\delta \sqrt{b}}, \quad \varepsilon=\frac{\sigma^{2}}{2 \delta}, \quad \text { and } \quad \rho=\frac{r}{\delta} .
$$

Setting $\sigma_{i}=\sigma$, as in the text, the state equation (2) can be written as:

$$
\mathrm{d} c_{i}=\left(-k_{i}-\beta k_{j}+\delta\right) c_{i} \mathrm{~d} t+c_{i} \sigma \mathrm{d} B_{i}
$$

In the new variables, the terms on the left and right side of the above equation take the form

$$
\begin{aligned}
\mathrm{d} c_{i} & =A \mathrm{~d} \tilde{c}_{i} \\
\left(-k_{i}-\beta k_{j}+\delta\right) c_{i} \mathrm{~d} t & =\left(-\frac{A}{\sqrt{b}} \tilde{k}_{i}-\beta \frac{A}{\sqrt{b}} \tilde{k}_{j}+\delta\right) A \tilde{c}_{i} \frac{1}{\delta} \mathrm{d} \tilde{t} \\
c_{i} \sigma \mathrm{d} B_{i} & =A \tilde{c}_{i} \sqrt{2 \delta \varepsilon} \frac{1}{\sqrt{\delta}} \mathrm{d} \tilde{B}_{i}
\end{aligned}
$$

Equation (33) in the scaled variables then reads as

$$
\mathrm{d} \tilde{c}_{i}=\left(1-\left(\tilde{k}_{i}+\beta \tilde{k}_{j}\right) \phi\right) \tilde{c}_{i} \mathrm{~d} \tilde{t}+\tilde{c}_{i} \sqrt{2 \varepsilon} \mathrm{d} \tilde{B}_{i} .
$$

The scaled instantaneous profit function takes the form

$$
\tilde{\pi}_{i}=\left(1-\tilde{q}_{i}-\tilde{q}_{j}-\tilde{c}_{i}\right) \tilde{q}_{i}-\tilde{k}_{i}^{2}
$$

Finally, observe that if $t=\tilde{t} / \delta$, then $e^{-\rho \tilde{t}}=e^{-r t}$ if and only if $\rho=r / \delta$. For notational convenience we omit tildes in the main text.

\section{B Boundary conditions}

To fully specify the system of coupled Hamilton-Jacobi-Bellman differential equations on a compact state space $\Omega$, we have specified the Neumann-type boundary conditions

$$
\frac{\partial U^{i}}{\partial \vec{n}}(s, x)=0
$$

for all points $x \in \partial \Omega$, and $\vec{n}$ being the outward pointing normal vector at $x$.

Consider the unrestricted problem in $\left(x_{1}, x_{2}\right)$ coordinates, and choose a fixed initial state $x(0)=$ $\left(x_{1}(0), x_{2}(0)\right)$ in the open set $\Omega$. The current time value function of a player equals

$$
V^{i}\left(x_{1}(0), x_{2}(0)\right)=\mathbb{E}_{0}\left(\int_{0}^{\tau}\left(g_{i}\left(x_{1}, x_{2}\right)-\left(\Gamma_{i}^{*}\right)^{2}\right) \mathrm{e}^{-\rho t} \mathrm{~d} t+\mathrm{e}^{-\rho \tau} V^{i}\left(x_{1}(\tau), x_{2}(\tau)\right)\right),
$$


where $\tau=\tau^{\varepsilon}$ is the (first) exit time from $\Omega$, and where the time dynamics are given as

$$
\mathrm{d} x_{i}=\left(\left(\Gamma_{i}^{*}+\beta \Gamma_{j}^{*}\right) \phi-1+\varepsilon\right) \mathrm{d} t-\sqrt{2 \varepsilon} \mathrm{d} B_{i} .
$$

Consider first the dynamics without the Brownian motion term. As the drift vector field is bounded, it follows that the exit time $\tau^{0}$ is bounded from below by some constant $c$, which is, for large values of $\underline{M}$ and $\bar{M}$, proportional to the distance $d$ of the initial state $x(0)$ to the boundary $\partial \Omega$ of $\Omega$.

For the full dynamics, including the Brownian motion term, the probability that $\tau^{\varepsilon}<\tau^{0} / 2$ is exponentially small in $d$. But the contribution of the 'boundary term' $\mathrm{e}^{-\rho \tau} V^{i}$ to the right hand side of (36) is then

$$
\begin{aligned}
\mathbb{E}_{0}\left(\mathrm{e}^{-\rho \tau} V^{i}(x(\tau))\right) & =\mathbb{E}_{0}\left(\mathrm{e}^{-\rho \tau} V^{i}(x(\tau)) \mid \tau \leq \frac{\tau^{0}}{2}\right)+\mathbb{E}_{0}\left(\mathrm{e}^{-\rho \tau} V^{i}(x(\tau)) \mid \tau>\frac{\tau^{0}}{2}\right) \\
& \leq \max _{x \in \partial \Omega}\left|V^{i}(x)\right| \mathbb{P}\left(\tau \leq \frac{\tau^{0}}{2}\right)+\mathrm{e}^{-\rho \tau^{0} / 2} \mathbb{E}_{0}\left(V^{i}(x(\tau)) \mid \tau>\frac{\tau^{0}}{2}\right) \\
& \leq c_{1} \mathrm{e}^{-c_{2} d} .
\end{aligned}
$$

This contribution constitutes the maximal error from misspecifying the boundary conditions. We see that it exponentially declines towards 0 as $|\bar{M}-\underline{M}| \rightarrow \infty$.

This is illustrated in Figure 14, which shows the policy function of firm 1 at the upper boundary of $x_{1}$ for three different values of $\bar{M}: \bar{M}=4.5, \bar{M}=5$, and $\bar{M}=5.5$, respectively. We see that consecutive solutions diverge only in the very close proximity of the boundary, where the solution corresponding to a smaller grid range sweeps sharply down to zero. Hence, by specifying a large enough range of the grid, we can always obtain a good approximation over the interior region of interest. This is further confirmed by the first plot in Figure 5, which shows that for a given large value of firm $j$ 's unit cost, the solution for firm $i$ converges to the deterministic monopoly solution as $\varepsilon \downarrow 0$.

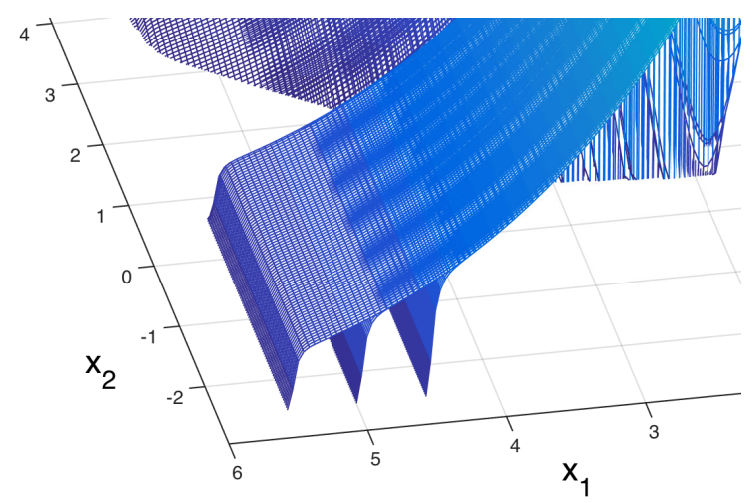

Figure 14: Solution at the boundary.

\section{References}

ACEMOgLu, D. And U. AKCIGit (2012): "Intellectual property rights policy, competition and innovation," Journal of the European Economic Association, 10, 1-42.

AMIR, M., R. AMIR, AND J. JIN (2000): "Sequencing R\&D decisions in a two-period duopoly with spillovers," Economic theory, 15, 297-317. 
AMIR, R. (2000): “Modelling imperfectly appropriable R\&D via spillovers," International journal of industrial organization, 18, 1013-1032.

Amir, R., I. EvstigneEv, AND J. Wooders (2003): "Noncooperative versus cooperative R\&D with endogenous spillover rates," Games and Economic Behavior, 42, 183-207.

ARROW, K. (1962): Economic welfare and the allocation of resources for invention, NBER, 609-626.

BAŞAR, T. AND G. J. OlSDER (1999): Dynamic Noncooperative Game Theory, Society for Industrial and Applied Mathematics.

Baily, M. N., C. Hulten, D. Campbell, T. Bresnahan, and R. E. Caves (1992): "Productivity dynamics in manufacturing plants," Brookings papers on economic activity. Microeconomics, 1992, $187-267$.

Benhabib, J., J. Perla, And C. Tonetti (2014): "Catch-up and fall-back through innovation and imitation," Journal of Economic Growth, 19, 1-35.

Benkard, C. L. (2000): "Learning and Forgetting: The Dynamics of Aircraft Production," American Economic Review, 90, 1034-1054.

Benveniste, L. AND J. Scheinkman (1982): "Duality theory for dynamic optimization models of economics: The continuous time case," Journal of Economic Theory, 27, 1-19.

Besanko, D., U. Doraszelski, Y. Kryukov, And M. SATterthwaite (2010): "Learning-byDoing, Organizational Forgetting, and Industry Dynamics," Econometrica, 78, 453-508.

Bhoovaraghavan, S., A. Vasudevan, And R. Chandran (1996): "Resolving the process vs. product innovation dilemma: A consumer choice theoretic approach," Management Science, 42, 232-246.

Bloom, N., M. Schankerman, And J. VAN REEnEn (2013): "Identifying technology spillovers and product market rivalry," Econometrica, 81, 1347-1393.

BLOOM, N. AND J. VAN REENEN (2011): "Human resource management and productivity," Handbook of labor economics, 4, 1697-1767.

BorkOVSKY, R. N., U. DORASZELSKI, AND Y. KRYUKOV (2012): "A dynamic quality ladder model with entry and exit: Exploring the equilibrium correspondence using the homotopy method," Quantitative Marketing and Economics, 10, 197-229.

CABRAL, L. AND M. H. RiORDAN (1997): “The learning curve, predation, antitrust, and welfare," The Journal of Industrial Economics, 45, 155-169.

CELlini, R. AND L. LAmbertini (2009): "Dynamic R\&D with spillovers: Competition vs cooperation," Journal of Economic Dynamics and Control, 33, 568-582.

Chen, J., U. Doraszelski, AND J. E. Harrington JR (2009): "Avoiding market dominance: Product compatibility in markets with network effects," The RAND Journal of Economics, 40, 455-485.

CRANDAll, M. G. AND P.-L. LiOns (1983): "Viscosity solutions of Hamilton-Jacobi equations," Transactions of the American Mathematical Society, 277, 1-42. 
D’Aspremont, C. AND A. JACQUEMIN (1988): "Cooperative and noncooperative R\&D in duopoly with spillovers," American Economic Review, 78, 1133-1137.

Dawid, H., M. Y. Keoula, M. Kopel, AND P. M. Kort (2015): "Product innovation incentives by an incumbent firm: A dynamic analysis," Journal of Economic Behavior \& Organization, 117, 411-438.

DE BONDT, R. (1997): "Spillovers and innovative activities," International Journal of Industrial Organization, 15, 1-28.

DIXIT, R. AND R. PINDYCK (2012): Investment under Uncertainty, Princeton University Press.

Dockner, E. AND F. WAGENER (2014): "Markov perfect Nash equilibria in models with a single capital stock," Economic Theory, 56, 585-625.

DORASZELSKI, U. AND K. L. JUDD (2012): "Avoiding the curse of dimensionality in dynamic stochastic games," Quantitative Economics, 3, 53-93.

Fowler, A. (1997): Mathematical Models in the Applied Sciences, Cambridge Texts in Applied Mathematics, Cambridge University Press.

Freidlin, M. I. AND A. D. Wentzell (1998): Random Perturbations of Dynamical Systems, 2nd Edition, Springer.

FURUKAWA, Y. (2007): "The protection of intellectual property rights and endogenous growth: Is stronger always better?" Journal of Economic Dynamics and Control, 31, 3644-3670.

Haurie, A., J. KrawczyK, And G. Zaccour (2012): Games and Dynamic Games, World Scientific.

Hinloopen, J., G. SMrkolu, And F. Wagener (2013): "From mind to market: A global, dynamic analysis of R\&D," Journal of Economic Dynamics and Control, 37, 2729-2754.

(2017): “R\&D Cooperatives and Market Collusion: A Global Dynamic Approach,” Tinbergen Institute Discussion Papers 17-020/VII, Tinbergen Institute.

Hounshell, D. A. (1988): Science and Corporate Strategy: Du Pont R\&D, 1902-1980, Cambridge University Press.

JUDD, K. L. (1998): Numerical Methods in Economics, Scientific and Engineering, MIT Press.

Kamien, M. I., E. Muller, AND I. ZANG (1992): "Research joint ventures and R\&D cartels," American Economic Review, 82, 1293-1306.

KATSOUlaCOS, Y. AND D. ULPH (1998): "Endogenous spillovers and the performance of research joint ventures," Journal of Industrial Economics, 46, 333-357.

Kloeden, P. E. And E. Platen (1992): Numerical Solution of Stochastic Differential Equations, Applications of mathematics : stochastic modelling and applied probability, Springer.

KÖNIG, M. D., J. LORENZ, AND F. ZiLibotTI (2016): "Innovation vs. imitation and the evolution of productivity distributions," Theoretical Economics, 11, 1053-1102. 
Kossioris, G., M. Plexousakis, A. Xepapadeas, A. de Zeeuw, and K. G. Maler (2008): "Feedback Nash equilibria for non-linear differential games in pollution control," Journal of Economic Dynamics and Control, 32, 1312-1331.

LUCAS JR, R. E. AND B. MOLL (2014): “Knowledge growth and the allocation of time," Journal of Political Economy, 122, 1-51.

Petit, M. L. AND B. TOLWINSKI (1999): “R\&D cooperation or competition?” European Economic Review, 43, 185-208.

Pollack, A. (1991): “One Day, Junior Got Too Big,” New York Times, Aug. 4, 6.

QIU, L. D. (1997): “On the dynamic efficiency of Bertrand and Cournot equilibria," Journal of economic theory, 75, 213-229.

SALANT, S. W. AND G. ShafFer (1999): "Unequal treatment of identical agents in Cournot equilibrium," American Economic Review, 89, 585-604.

SCHIESSER, W. E. (1991): The Numerical Method of Lines: Integration of Partial Differential Equations, Academic Press.

Shenkar, O. (2010): Copycats: How Smart Companies Use Imitation to Gain a Strategic Edge, Harvard Business Review Press.

SMrkolJ, G. AND F. WAGener (2016): "Dynamic R\&D with spillovers: A comment," Journal of Economic Dynamics and Control, 73, 453-457.

Utterback, J. M. (1994): Mastering the Dynamics of Innovation. How Companies Can Seize Opportunities in the Face of Technological Change, Harvard Business School Press.

VON HIPPEL, E. (1988): The Sources of Innovation, New York: Oxford University Press. 